\title{
PIECEWISE POLYNOMIAL COLLOCATION FOR BOUNDARY INTEGRAL EQUATIONS*
}

\author{
KENDALL E. ATKINSON ${ }^{\dagger}$ AND DAVID CHIEN ${ }^{\ddagger}$
}

\begin{abstract}
This paper considers the numerical solution of boundary integral equations of the second kind for Laplace's equation $\Delta u=0$ on connected regions $D$ in $\mathbf{R}^{3}$ with boundary $S$. The boundary $S$ is allowed to be smooth or piecewise smooth, and we let $\left\{\Delta_{K} \mid 1 \leq K \leq N\right\}$ be a triangulation of $S$. The numerical method is collocation with approximations which are piecewise quadratic in the parametrization variables, leading to a numerical solution $u_{N}$. Superconvergence results for $u_{N}$ are given for $S$ a smooth surface and for a special type of refinement strategy for the triangulation. We show that $u-u_{N}$ is $O\left(\delta^{4} \log \delta\right)$ at the collocation node points, with $\delta$ being the mesh size for $\left\{\Delta_{K}\right\}$. Error analyses are given are given for other quantities, and an important error analysis is given for the approximation of $S$ by piecewise quadratic interpolation on each triangular element, with $S$ either smooth or piecewise smooth. The convergence result we prove is only $O\left(\delta^{2}\right)$ but the numerical experiments suggest the result is $O\left(\delta^{4}\right)$ for the error at the collocation points, especially when $S$ is a smooth surface. The numerical integration of the collocation integrals is discussed, and extended numerical examples are given for problems involving both smooth and piecewise smooth surfaces.
\end{abstract}

Key words. integral equations, quadrature interpolation, Laplace's equation, numerical integration

AMS subject classifications. 65R20, 35J05, 45L10, 65D05, 65D30

1. Introduction. In this paper, we consider the numerical solution of boundary integral equations of the second kind for solving Laplace's equation $\Delta u=0$ on connected regions $D$ in $\mathbf{R}^{3}$. The collocation method with piecewise polynomial approximations is the numerical method being analyzed. Because of the practical need to use easily computable approximations of the surface, we analyze the effect of using interpolation to approximate the surface of the region. We also discuss the effect of numerical integration of the collocation integrals.

A major consideration in the error analysis of numerical methods for these boundary integral equations is whether the boundary of $D$, call it $S$, is smooth or piecewise smooth. If $S$ is smooth, then the associated integral operator is compact and there is a wealth of results available for the error analysis. But if $S$ is only piecewise smooth, then the integral operator is not compact, and moreover, the operator can be viewed as involving a Dirac delta function in its definition. In this case, other methods of error analysis are required. The most widely used techniques originated with Wendland [23], in which he adapted and greatly extended a technique introduced in [20] for the theoretical analysis of such integral equations for the planar Dirichlet problem for Laplace's equation. We use Wendland's ideas in our analysis of the collocation method given below in $\S 5$. Other approaches for this case are under development; for example, see Elschner [10] in which results of Graham and Chandler [12] for the planar problem are generalized to Galerkin methods for polyhedral boundaries in $\mathbf{R}^{3}$, and see Rathsfeld [17]. For a general survey of the use of boundary integral equations to solve Laplace's equation in three dimensions, see [5].

Two problems for Laplace's equation and their associated boundary integral equations are studied in this paper.

P1. The interior Dirichlet problem. Let $D$ be a bounded, open, simply connected region in $\mathbf{R}^{3}$ and let its boundary $S$ be piecewise smooth, which is defined more precisely in $\S 2$. The problem is to find $u \in C(\bar{D}) \cap C^{2}(D)$ such that

\footnotetext{
${ }^{*}$ Received by the editors August 19, 1992; accepted for publication (in revised form) March 14, 1994.

†Department of Mathematics, University of Iowa, Iowa City, Iowa 52242 (atkinson@math . ui iowa . edu). The research of this author was supported in part by National Science Foundation grant DMS-9003287.

$\ddagger$ Department of Mathematics, California State University San Marcos, San Marcos, California 92096 (chien@ csusm.edu).
} 


$$
\begin{aligned}
\Delta u(A) & =0, \quad A \in D, \\
u(P) & =f(P), \quad P \in S .
\end{aligned}
$$

We assume $u$ can be represented as a double-layer potential:

$$
u(A)=\int_{S} \rho(Q) \frac{\partial}{\partial v_{Q}}\left[\frac{1}{|A-Q|}\right] d S_{Q}, \quad A \in D .
$$

The density function $\rho$ is determined from the integral equation

(2) $2 \pi \rho(P)+\int_{S} \rho(Q) \frac{\partial}{\partial \nu_{Q}}\left[\frac{1}{|P-Q|}\right] d S_{Q}+[2 \pi-\Omega(P)] \rho(P)=f(P), \quad P \in S$.

$v_{Q}$ denotes the unit normal to $S$ at $Q$ (if it exists), pointing into $D$. The quantity $\Omega(P)$ is the inner solid angle of $S$ at $P \in S$ and we assume

$$
0<\Omega(P)<4 \pi .
$$

Symbolically, we write the integral equation (2) as

$$
(2 \pi+\mathcal{K}) \rho=f .
$$

Under suitable assumptions on $S$,

$$
\mathcal{K}: C(S) \rightarrow C(S)
$$

is a bounded linear operator.

P2. The exterior Neumann problem. Let $D$ and $S$ be as above and let $D_{e}=\mathbf{R}^{3} \backslash \bar{D}$, the region exterior to $D$ and $S$. The problem is to find $u \in C\left(\bar{D}_{e}\right) \cap C^{2}\left(D_{e}\right)$ such that

$$
\begin{gathered}
\Delta u(A)=0, \quad A \in D_{e}, \\
\frac{\partial u(P)}{\partial v_{P}}=f(P), \quad P \in S, \\
u(P)=O\left(|P|^{-1}\right),|\nabla u(P)|=O\left(|P|^{-2}\right) \text { as }|P| \rightarrow \infty .
\end{gathered}
$$

It can be shown that such a function $u$ exists (under suitable assumptions on $S$ and $f$ ) and that Green's third identity can be applied to $u$ :

(4) $4 \pi u(A)=\int_{S} f(Q) \frac{1}{|A-Q|} d S_{Q}-\int_{S} u(Q) \frac{\partial}{\partial v_{Q}}\left[\frac{1}{|A-Q|}\right] d S_{Q}, \quad A \in D$.

To find $u$ on $S$, we solve the integral equation

$$
\begin{aligned}
2 \pi u(P) & +\int_{S} u(Q) \frac{\partial}{\partial v_{Q}}\left[\frac{1}{|P-Q|}\right] d S_{Q}+[2 \pi-\Omega(P)] u(P) \\
& =\int_{S} f(Q) \frac{1}{|P-Q|} d S_{Q}, \quad P \in S .
\end{aligned}
$$

Then (4) gives $u$ on $D_{e}$. Symbolically, we write (5) as

$$
(2 \pi+\mathcal{K}) u=\mathcal{S} f
$$

with $\mathcal{K}$ as before and $\mathcal{S}$ the single-layer potential integral operator. 
The integral equations (2) and (5) are different only in their right-hand inhomogeneous term. With (5), we can study the error in the numerical solution of the integral equation by using problems for which we know the true solution of (3). With equation (2), we do not know the true solution in general (except when $f \equiv 1$ ), and thus the numerical solution must be checked indirectly by evaluating (1) numerically and comparing it to a known solution $u$. This turns out to also be of interest, because integral formulas like (1) are generally known to converge faster than the density function that solves the integral equation. A further discussion is given later.

In $\S 2$, we describe briefly the triangulation of the surface $S$. The collocation method and the surface approximation are based on piecewise quadratic isoparametric interpolation; this is described in $\S 2$, together with the numerical integration methods used in evaluating the collocation integrals. The collocation method with $S$ smooth is discussed in $\S 3$, and numerical examples are given in $\S 4$. The corresponding results for the collocation method when $S$ is only piecewise smooth are given in $\S \S 5$ and 6. Some of the methods of this paper follow those of Atkinson [2], [3], but we also involve the new methods of analysis given in Chien [8] to improve on the error results of the earlier papers.

Although our analysis is for only quadratic approximation, the method used will generalize to other degrees of piecewise polynomial approximation. The difficulty of our argument has led us to specialize to one case; in addition, it is one of the more important cases.

2. Preliminaries. We describe the triangulation scheme and associated interpolation and quadrature. The method used was discussed in [2], [3], and we assume a familiarity with those papers, including the notation used in them.

As discussed in [2], we assume the surface $S$ can be written as

$$
S=S_{1} \cup S_{2} \cup \cdots \cup S_{J},
$$

where each $S_{i}$ is a closed, "smooth" surface in $\mathbf{R}^{3}$. The only possible intersection of a pair $S_{i}$ and $S_{j}$ is to be along a common portion of the edges of these two sub-surfaces. Assume that for each $S_{j}$, there is a mapping

$$
F_{j}: R_{j} \underset{\text { onto }}{\stackrel{1-1}{\longrightarrow}} S_{j}, \quad 1 \leq j \leq J,
$$

where $R_{j}$ is a polygonal domain in the plane and $F_{j} \in C^{6}\left(R_{j}\right)$. In this case, we say $S$ is piecewise smooth. By a smooth surface, we mean that for each point $P \in S$, there is a neighborhood on $S$ of $P$, with the neighborhood having a local six-times continuously differentiable parametrization in $\mathbf{R}^{2}$.

The surface $S$ of (6) is divided into a triangular mesh

$$
\left\{\Delta_{K, N} \mid 1 \leq K \leq N\right\}
$$

for a sequence $N=N_{1}, N_{2}, \ldots$ Each $S_{j}$ is to be broken apart into a set of nonoverlapping triangular shaped elements $\Delta_{K, N_{j}}$ 's, about which we say more below. In referring to the element $\Delta_{K, N}$, the reference to $N$ will be omitted, but understood implicitly. Define the mesh size of (8) by

$$
\delta_{N}=\max _{1 \leq K \leq N} \operatorname{diam}\left(\Delta_{K}\right)
$$

$$
\operatorname{diam}\left(\Delta_{K}\right)=\max _{p, q \in \Delta_{K}}|p-q| .
$$

Let $\sigma$ denote the unit simplex in the st-plane

$$
\sigma=\{(s, t) \mid 0 \leq s, t, s+t \leq 1\} .
$$




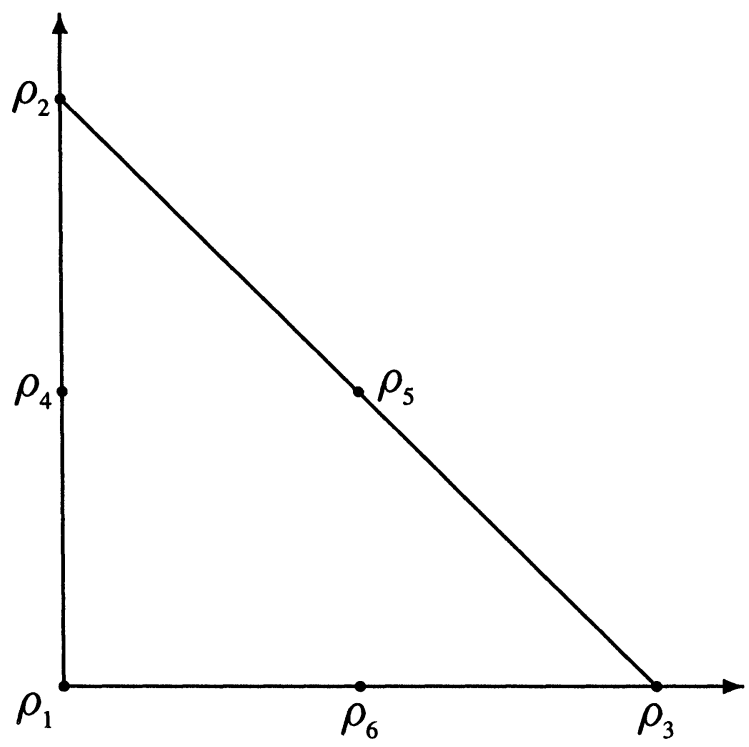

FIG. 1. The unit simplex.

Let $\rho_{1}, \ldots, \rho_{6}$ denote the three vertices and three midpoints of the sides of $\sigma$, numbered according to Fig. 1.

One way of obtaining the triangulation (8) and the mappings from $\sigma$ to each $\Delta_{K}$ is by means of the parametric representation (7) for the region $S_{j}$ of (6). Triangulations of $R_{j}$ map onto triangulations of $S_{j}$. Since the $R_{j}$ 's are polygonal domains and can be written as a union of triangles, without loss of generality, we assume in this paper that the $R_{j}$ 's are triangles. A paraboloid with a top is a good example of an $S$ that satisfies our assumptions; a circular cone is an example of an $S$ for which some of above assumptions are not valid, because of the discontinuity of the gradient at the vertex. Define

Let $\widehat{\Delta}_{K}$ be an element in the triangulation of $R_{j}$, and let $\widehat{v}_{1}, \widehat{v}_{2}$, and $\widehat{v}_{3}$ be its vertices.

$$
m_{K}(s, t)=F_{j}\left(u \widehat{v}_{1}+t \widehat{v}_{2}+s \widehat{v}_{3}\right), \quad u=1-s-t, \quad(s, t) \in \sigma
$$

and let $\Delta_{K}$ be the image of $\widehat{\Delta}_{K}$ under this mapping. Also, if any two elements in this triangulation have a side in common, then their intersection will be an entire side of both triangles. Most surfaces $S$ of interest can be decomposed as in (6), with each $S_{j}$ representable as in (7). Also, the surface $S$ could be smooth, and we would often still want to decompose it as in (6).

The mapping (10) is used in defining interpolation and numerical integration on $\Delta_{K}$. Introduce the node points for $\Delta_{K}$ by

$$
v_{j, K}=m_{K}\left(\rho_{j}\right), \quad j=1, \ldots, 6 .
$$

Collectively, the node points of the triangulation $\left\{\Delta_{K}\right\}$ will be denoted by

$$
\left\{v_{i} \mid 1 \leq i \leq N_{v}\right\}
$$

with $N_{v}$ being the number of distinct node points. 


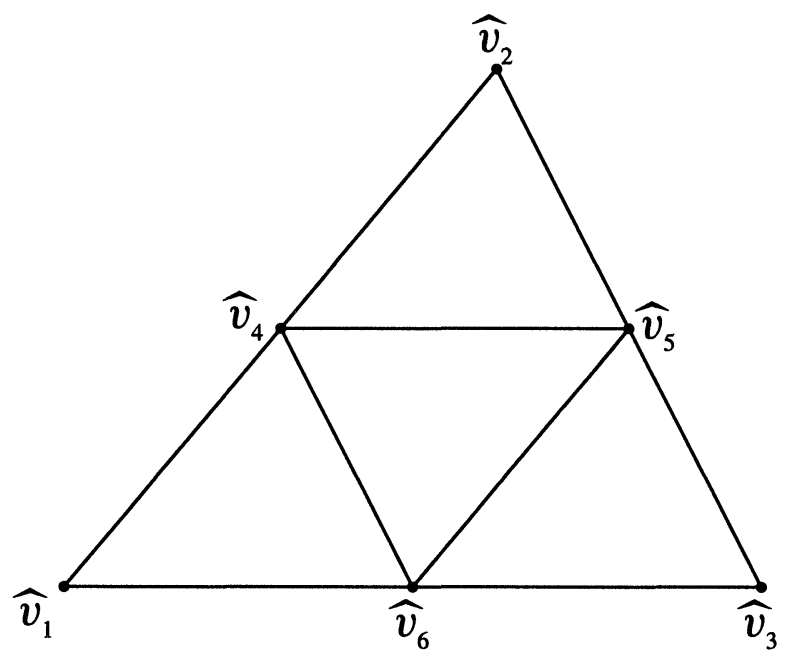

FIG. 2. Refinement.

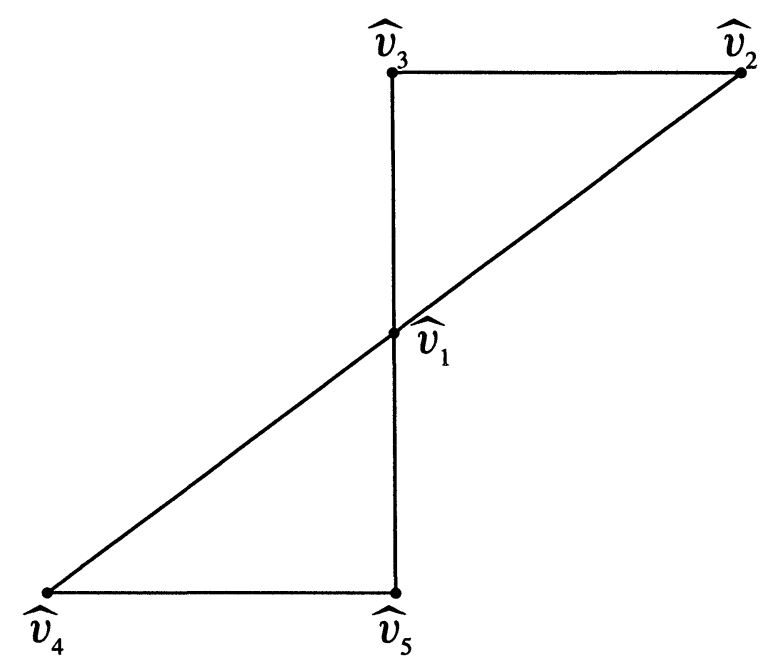

FIG. 3. A symmetric pair of triangles.

The sequence of triangulations (8) will usually be obtained by successive refinements. The refinement process is based on connecting the midpoints of the sides of a given element $\widehat{\Delta}_{K}$. Given $\left\{\widehat{v}_{1}, \ldots, \widehat{v}_{6}\right\}$, connect $\widehat{v}_{4}, \widehat{v}_{5}, \widehat{v}_{6}$ by straight lines, as in Fig. 2, producing four new triangular elements. The new elements all are congruent and they are similar to $\widehat{\Delta}_{K}$. More importantly, any symmetric pair of triangles, as shown in Fig. 3, has the following property:

$$
\widehat{v}_{1}-\widehat{v}_{2}=-\left(\widehat{v}_{1}-\widehat{v}_{4}\right) \text { and } \widehat{v}_{1}-\widehat{v}_{3}=-\left(\widehat{v}_{1}-\widehat{v}_{5}\right) \text {. }
$$

The assumptions on $S$ and the node points that we made in this section are for the use of quadratic interpolation. There are other degrees of interpolation that can be used, and the assumptions on the smoothness of $S$ and the definition of the nodes will change appropriately. But the general process of refinement will still remain the same and we still subdivide $\Delta_{K}$ 's in the same way as for the quadratic interpolation. 
To define interpolation, introduce the six basis functions for quadratic interpolation on $\sigma$. Letting $u=1-(s+t)$, define

$$
\begin{array}{lll}
l_{1}(s, t)=u(2 u-1), & l_{2}(s, t)=t(2 t-1), & l_{3}(s, t)=s(2 s-1), \\
l_{4}(s, t)=4 t u, & l_{5}(s, t)=4 s t, & l_{6}(s, t)=4 s u .
\end{array}
$$

Define a corresponding set of basis functions $\left\{l_{j, K}(q)\right\}$ on $\Delta_{K}$ :

$$
l_{j, K}\left(m_{K}(s, t)\right)=l_{j}(s, t), \quad 1 \leq j \leq 6, \quad 1 \leq K \leq N .
$$

Given a function $f \in C(S)$, define

$$
\mathcal{P}_{N} f(q)=\sum_{j=1}^{6} f\left(v_{j, K}\right) l_{j, K}(q), \quad q \in \Delta_{K},
$$

for $K=1, \ldots, N$. This is called the piecewise quadratic isoparametric function interpolating $f$ on the nodes of the mesh $\Delta_{K}$ for $S$.

It is straightforward that $\mathcal{P}_{N}$ is a bounded projection operator and $\left\|\mathcal{P}_{N}\right\|=5 / 3$. Also, for any $f \in C^{3}(S)$,

$$
\left\|f-\mathcal{P}_{N} f\right\|_{\infty}=O\left(\widehat{\delta}_{N}^{3}\right)
$$

where $\widehat{\delta}_{N}$ is the mesh size of the triangulation $\left\{\widehat{\Delta}_{K, N}\right\}$ of $R_{j}$ 's. See [2].

Other kinds of interpolation can be used, such as piecewise cubic isoparametric interpolation. In this case, we need ten node points, $\rho_{1}, \ldots, \rho_{10}$, and ten basis functions for the interpolation on $\sigma$. The error analysis is the same, although somewhat more complicated.

We also use the same quadratic interpolation scheme to construct an approximate surface $\widetilde{S}$ for $S$. The approximate surface $\widetilde{S}$ is composed of elements $\widetilde{\Delta}_{1}, \ldots, \widetilde{\Delta}_{K}$, with $\widetilde{\Delta}_{K}$ an interpolant of $\Delta_{K}$. Write

$$
m_{K}(s, t)=\left[\begin{array}{c}
x_{K}^{1}(s, t) \\
x_{K}^{2}(s, t) \\
x_{K}^{3}(s, t)
\end{array}\right], \quad(s, t) \in \sigma .
$$

The reference to $K$ will be omitted, but understood implicitly. Define

(15) $\tilde{m}_{K}(s, t)=\sum_{j=1}^{6} m_{K}\left(\rho_{j}\right) l_{j}(s, t)=\left[\begin{array}{c}\sum_{j=1}^{6} x_{K}^{1}\left(\rho_{j}\right) l_{j}(s, t) \\ \sum_{j=1}^{6} x_{K}^{2}\left(\rho_{j}\right) l_{j}(s, t) \\ \sum_{j=1}^{6} x_{K}^{3}\left(\rho_{j}\right) l_{j}(s, t)\end{array}\right], \quad(s, t) \in \sigma$

Thus, $\tilde{m}_{K}(s, t)$ interpolates $m_{K}(s, t)$ at $\left\{\rho_{1}, \ldots, \rho_{6}\right\}$, and each component is quadratic in $(s, t)$.

We introduce two major numerical integration schemes that we have used. The first numerical integration method is the 3-point rule

$$
\int_{\sigma} h(s, t) d \sigma \approx \frac{1}{6} \sum_{j=4}^{6} h\left(\rho_{j}\right)
$$

This method has degree of precision two, integrating exactly all quadratic polynomials. Chien [8] shows that the associated composite rule over $S$ is $O\left(\widehat{\delta}_{N}^{4}\right)$ where $\widehat{\delta}_{N}$ is the mesh size of $\left\{\widehat{\Delta}_{K}\right\}$. 
The 3-point rule is mainly for computing integrals if the integrands are continuous. In order to get the above results, the integrands are required to be four times continuously differentiable. If the integrands are continuous or smooth on the $\Delta_{K}$, but there is a nearby singularity, we need to use a better numerical integration method. The second method is the rule T2:5-1 from Stroud [22, p. 314]:

$$
\int_{\sigma} h(s, t) d \sigma \approx \sum_{j=1}^{7} w_{j} h\left(r_{j}\right) .
$$

The weights $w_{j}$ and nodes $r_{j}$ are given in the above reference. This formula has degree of precision five.

3. Collocation on smooth boundaries. Our collocation method for solving an integral equation $(\lambda+\mathcal{K}) \rho=g$ can be written as

$$
\left(\lambda+\mathcal{P}_{N} \mathcal{K}\right) \rho_{N}=\mathcal{P}_{N} g, \quad \lambda=2 \pi .
$$

The function $g$ can be the function $f$ of (2) or $\mathcal{S} f$ of (5). We discuss results for this approximation and later in the section give error results for the effect of using an interpolatory approximation of the smooth surface $S$.

An important auxiliary solution for the collocation method is the iterated collocation solution

$$
\hat{\rho}_{N}=\frac{1}{\lambda}\left(g-\mathcal{K} \rho_{N}\right)
$$

It satisfies the equations

$$
\begin{aligned}
\left(\lambda+\mathcal{K} \mathcal{P}_{N}\right) \hat{\rho}_{N} & =g, \\
\mathcal{P}_{N} \hat{\rho}_{N} & =\rho_{N} .
\end{aligned}
$$

The questions of stability for (18) and (19) are linked by the identities

$$
\begin{aligned}
& \left(\lambda+\mathcal{K} \mathcal{P}_{N}\right)^{-1}=\frac{1}{\lambda}\left[I-\mathcal{K}\left(\lambda+\mathcal{P}_{N} \mathcal{K}\right)^{-1} \mathcal{P}_{N}\right] \\
& \left(\lambda+\mathcal{P}_{N} \mathcal{K}\right)^{-1}=\frac{1}{\lambda}\left[I-\mathcal{P}_{N}\left(\lambda+\mathcal{K} \mathcal{P}_{N}\right)^{-1} \mathcal{K}\right]
\end{aligned}
$$

The solvability of (18) is determined from the standard theory for projection methods, for example, see Atkinson [1, pp. 50-62]. With the assumption of (a) compactness for $\mathcal{K}$ : $C(S) \rightarrow C(S)$, and (b) pointwise convergence on $C(S)$ of the projections $\mathcal{P}_{N}$ to $I$, we have that

$$
\left\|\left(I-\mathcal{P}_{N}\right) \mathcal{K}\right\| \rightarrow 0 \text { as } n \rightarrow \infty .
$$

From this, we have the standard result that if $(\lambda+\mathcal{K})^{-1}$ exists on $C(S)$, then $\left(\lambda+\mathcal{P}_{N} \mathcal{K}\right)^{-1}$ exists and is uniformly bounded for all sufficiently large $N$, say $N \geq N_{0}$. The existence of uniform boundedness of $\left(\lambda+\mathcal{K} \mathcal{P}_{N}\right)^{-1}$ then follows from (21).

For the error in $\rho_{N}$ and $\hat{\rho}_{N}$, use

$$
\begin{aligned}
& \rho-\rho_{N}=\lambda\left(\lambda+\mathcal{P}_{N} \mathcal{K}\right)^{-1}\left(\rho-\mathcal{P}_{N} \rho\right), \\
& \rho-\hat{\rho}_{N}=-\left(\lambda+\mathcal{K} \mathcal{P}_{N}\right)^{-1} \mathcal{K}\left(\rho-\mathcal{P}_{N} \rho\right) .
\end{aligned}
$$


The quantity $\mathcal{K}\left(\rho-\mathcal{P}_{N} \rho\right)$ often converges to zero more rapidly than $\rho-\mathcal{P}_{N} \rho$ does. Using (20), we conclude that $\rho_{N}$ is superconvergent to $\rho$ at the collocation node points. We make use of this in the following theorem.

THEOREM 3.1. Consider the integral equation (2) and (5) with solution $\rho$. Let $S$ be a smooth surface in $\mathbf{R}^{3}$ and assume the unknown function $\rho \in C^{4}(S)$. Then

$$
\max _{1 \leq i \leq N_{v}}\left|\rho\left(v_{i}\right)-\rho_{N}\left(v_{i}\right)\right|=O\left(\widehat{\delta}_{N}^{4} \log \widehat{\delta}_{N}\right),
$$

where $\widehat{\delta}_{N}$ is the mesh size of the triangulation $\left\{\widehat{\Delta}_{K, N}\right\}$ of the $R_{j}$ 's.

Proof. (a) The major part of the proof is concerned with measuring $\mathcal{K}\left(I-\mathcal{P}_{N}\right) \rho(P)$ for all $P=v_{i}$, a node point. Later in the proof, we use this to prove (22). Note we use the exact surface $S$ in this theorem. Since the solid angle $\Omega(P)=2 \pi$ for every $P$ on a smooth surface, the integral equation (2) can be simplified as

$$
2 \pi \rho(P)+\int_{S} \rho(Q) \frac{\partial}{\partial \nu_{Q}}\left[\frac{1}{|P-Q|}\right] d S_{Q}=f(P), \quad P \in S .
$$

Using the triangulation scheme in $\S 2$, the compact operator $\mathcal{K}$ can be written as

$$
\mathcal{K} \rho(P)=\sum_{K} \int_{\sigma} \rho\left(m_{K}(s, t)\right) \frac{\partial}{\partial \nu_{Q}}\left[\frac{1}{\left|P-m_{K}(s, t)\right|}\right]\left|D_{s} m_{K} \times D_{t} m_{K}\right| d \sigma .
$$

For $Q=m_{K}(s, t)$,

$$
v(s, t)=v_{Q}= \pm \frac{D_{s} m_{K} \times D_{t} m_{K}}{\left|D_{s} m_{K} \times D_{t} m_{K}\right|},
$$

with the sign chosen so that $\nu_{Q}$ points into the bounded domain $D$.

Without loss of generality, we assume the sign of $v_{Q}$ is always positive, and (23) becomes

$$
\mathcal{K} \rho(P)=\sum_{K} \int_{\sigma} \rho\left(m_{K}(s, t)\right) \frac{\left(D_{s} m_{K} \times D_{t} m_{K}\right) \cdot\left(P-m_{K}(s, t)\right)}{\left|P-m_{K}(s, t)\right|^{3}} d s d t .
$$

In order to measure the error $\mathcal{K}\left(I-\mathcal{P}_{N}\right) \rho(P)$ for $P$ a node point, we need to examine the local error contributed by each $\Delta_{K}$.

For each $\Delta_{K}$, the integrand of the equation (24) has one singularity at $P$ when $P \in \Delta_{K}$, and it is smooth over $\Delta_{K}$ with $P \notin \Delta_{K}$, although it is increasingly peaked as $P$ and $\Delta_{K}$ become closer together. We first compute the error for those $\Delta_{K}$ 's which contain $P$.

For simplifying notation, we assume $P=(0,0,0)$ and $m_{K}(0,0)=(0,0,0)$. The error in integrating over $\Delta_{K}$ equals

$$
\int_{\sigma}\left(\rho\left(m_{K}(s, t)\right)-\mathcal{P}_{N} \rho\left(m_{K}(s, t)\right)\right) \frac{\left(D_{s} m_{K} \times D_{t} m_{K}\right) \cdot m_{K}(s, t)}{\left|m_{K}(s, t)\right|^{3}} d s d t .
$$

This integral exists even though $\left|m_{K}(0,0)\right|=0$. To see this, use the Taylor error formula for the $x_{K}^{i}$ about $(s, t)=(0,0)$. Then (25) equals

$$
\int_{\sigma} \frac{h\left(s, t ; \widehat{\delta}^{7}\right)}{g\left(s, t ; \widehat{\delta}^{3}\right)} d \sigma
$$

where $h$ and $g$ are polynomials in $s$ and $t$, and their coefficients are of size $O\left(\widehat{\delta}^{7}\right)$ and $O\left(\widehat{\delta}^{3}\right)$, respectively. Also, $h$ and $g$ are polynomials of degrees two and three, respectively, which shows 
the existence of integral (26), which is $O\left(\widehat{\delta}^{4}\right)$. (When speaking of an order of convergence, say one based on $\widehat{\delta}$, the order of convergence is uniform with respect to any absent variable or index.)

When $P \notin \Delta_{K}, P-m_{K}$ never equals zero for $(s, t) \in \sigma$. The kernel function, $\kappa(s, t)$,

$$
\kappa(s, t)=\frac{\left(D_{s} m_{K} \times D_{t} m_{K}\right) \cdot\left(P-m_{K}(s, t)\right)}{\left|P-m_{K}(s, t)\right|^{3}}
$$

is smooth. Compute the partial derivative $\kappa_{s}$ before expanding $\kappa(s, t)$ about $(0,0)$.

$$
\begin{aligned}
\kappa_{s}(s, t)= & \frac{\left[D_{s}\left(D_{s} m_{K} \times D_{t} m_{K}\right)\right] \cdot\left(P-m_{K}\right)-\left(D_{s} m_{K} \times D_{t} m_{K}\right) \cdot D_{s} m_{K}}{\left|P-m_{K}\right|^{3}} \\
& -3 \frac{\left[\left(D_{s} m_{K} \times D_{t} m_{K}\right) \cdot\left(P-m_{K}\right)\right]\left[D_{s} m_{K} \cdot\left(P-m_{K}\right)\right]}{\left|P-m_{K}\right|^{5}} \\
= & \frac{\left[D_{s}\left(D_{s} m_{K} \times D_{t} m_{K}\right)\right] \cdot\left(P-m_{K}\right)}{\left|P-m_{K}\right|^{3}} \\
& -3 \frac{\left[\left(D_{s} m_{K} \times D_{t} m_{K}\right) \cdot\left(P-m_{K}\right)\right]\left[D_{s} m_{K} \cdot\left(P-m_{K}\right)\right]}{\left|P-m_{K}\right|^{5}} .
\end{aligned}
$$

The term $\left(D_{s} m_{K} \times D_{t} m_{K}\right) \cdot D_{s} m_{K}$ was dropped because $\left(D_{s} m_{K} \times D_{t} m_{K}\right) \perp D_{s} m_{K}$. Also

$$
\left|\left(D_{s} m_{K} \times D_{t} m_{K}\right) \cdot\left(P-m_{K}\right)\right|=\left|D_{s} m_{K} \times D_{t} m_{K}\right| \cdot\left|P-m_{K}\right| \cdot|\cos \theta| .
$$

$\theta$ is the angle between the vectors $D_{s} m_{K} \times D_{t} m_{K}$ and $P-m_{K}$, and $\theta$ is a function of $s$ and $t$ :

$$
|\cos \theta| \leq\left|P-m_{K}(s, t)\right| \cdot \text { constant } \quad \forall(s, t) .
$$

See [16, p. 349]. Therefore, $\kappa_{s}$ is $O\left(\widehat{\delta}^{3} / d_{K}^{2}\right)$ where $d_{K}=\left|P-m_{K}(0,0)\right|$. Using a similar calculation, we find that $\kappa_{t}$ is also $O\left(\widehat{\delta}^{3} / d_{K}^{2}\right)$. We now expand $\kappa(s, t)$ about $(0,0)$ and have the following formula:

$$
\kappa(s, t)=\kappa(0,0)+O\left(\widehat{\delta}^{3} / d_{K}^{2}\right) .
$$

The error of $\rho\left(m_{K}\right)-\mathcal{P}_{N} \rho\left(m_{K}\right)$ is

$$
\rho\left(m_{K}\right)-\mathcal{P}_{N} \rho\left(m_{K}\right)=H_{K}(s, t)+O\left(\widehat{\delta}^{4}\right),
$$

where

$$
H_{K}(s, t)=\frac{1}{3 !}\left[\left(s \frac{\partial}{\partial s}+t \frac{\partial}{\partial t}\right)^{3} x^{i}(0,0)-\sum_{j=1}^{6}\left(s_{j} \frac{\partial}{\partial s}+t_{j} \frac{\partial}{\partial t}\right)^{3} x^{i}(0,0) l_{j}(s, t)\right] .
$$

Note that $\kappa(0,0)$ and $H_{K}(s, t)$ are $O\left(\widehat{\delta}^{2} / d_{K}^{2}\right)$ and $O\left(\widehat{\delta}^{3}\right)$, respectively.

Combining (28) and (29), we have

$$
\begin{aligned}
\int_{\sigma} \kappa(s, t)\left(\rho\left(m_{K}\right)-\mathcal{P}_{N} \rho\left(m_{K}\right)\right) d \sigma & =\int_{\sigma}\left(\kappa(0,0)+O\left(\frac{\widehat{\delta}^{3}}{d_{K}^{2}}\right)\right) H_{K}(s, t) d \sigma \\
& =O\left(\frac{\widehat{\delta}^{5}}{d_{K}^{2}}\right)+O\left(\frac{\widehat{\delta}^{6}}{d_{K}^{2}}\right)
\end{aligned}
$$

for every $\Delta_{K}$ which does not contain $P$. 
We now add all errors contributed by each $\Delta_{K}$. Let $T^{\prime}$ be the set of $\Delta_{K}$ 's which contain $P$, and let $T$ be the set of the remaining $\Delta_{K}$ 's, which do not contain $P$. Then,

$$
\begin{aligned}
\mathcal{K}\left(\rho-P_{n} \rho\right)(P)= & \int_{S} \kappa(P, Q)\left(\rho(Q)-\mathcal{P}_{N} \rho(Q)\right) d S_{Q} \\
= & \sum_{\Delta_{K} \in T^{\prime}} \int_{\sigma} \kappa(s, t)\left(\rho\left(m_{K}\right)-\mathcal{P}_{N} \rho\left(m_{K}\right)\right) d \sigma \\
& +\sum_{\Delta_{K} \in T} \int_{\sigma} \kappa(s, t)\left(\rho\left(m_{K}\right)-\mathcal{P}_{N} \rho\left(m_{K}\right)\right) d \sigma \\
= & O\left(\widehat{\delta}^{4}\right)+\sum_{\Delta_{K} \in T} \int_{\sigma} \kappa(s, t)\left(\rho\left(m_{K}\right)-\mathcal{P}_{N} \rho\left(m_{K}\right)\right) d \sigma
\end{aligned}
$$

$O\left(\widehat{\delta}^{4}\right)$ is contributed by $\Delta_{K}$ 's which are in $T^{\prime}$, and $T^{\prime}$ has at most six elements.

The error contributed by each $\Delta_{K}$ in $T$ is $O\left(\widehat{\delta}^{5} / d_{K}^{2}\right)$. Examining the error carefully, we find that cancellation happens on each symmetric pair of triangles. Thus, for the dominant terms in the error,

$$
\kappa(0,0) H_{i}(s, t)+\kappa(0,0) H_{j}(s, t)=0
$$

if $\Delta_{i}$ and $\Delta_{j}$ are a symmetric pair of triangles. This improves the error from $O\left(\widehat{\delta}^{5} / d_{K}^{2}\right)$ to $O\left(\widehat{\delta}^{6} / d_{K}^{2}\right)$ for each $\Delta_{K}$ that is part of a symmetric pair of triangles. Let $T_{1}$ be the set of these kinds of triangles. Let $T_{2}$ be the set of triangles that are not in $T_{1}$.

The error contributed by triangles in $T_{2}$ arises from the term

$$
\begin{aligned}
\left|\kappa(0,0) \cdot H_{K}(s, t)\right| \\
\quad=\left|\frac{\left(D_{s} m_{K}(0,0) \times D_{t} m_{K}(0,0)\right) \cdot\left(P-m_{K}(0,0)\right)}{\left|P-m_{K}(0,0)\right|^{3}}\right|\left|H_{K}(s, t)\right| \\
\quad=\left|\frac{\left|\left(D_{s} m_{K}(0,0) \times D_{t} m_{K}(0,0)\right)\right| \cdot\left|\left(P-m_{K}(0,0)\right)\right| \cdot \cos \theta}{\left|P-m_{K}(0,0)\right|^{3}}\right|\left|H_{K}(s, t)\right| \\
\quad \leq \frac{\left|\left(D_{s} m_{K}(0,0) \times D_{t} m_{K}(0,0)\right)\right|}{\left|P-m_{K}(0,0)\right|}\left|H_{K}(s, t)\right|=O\left(\frac{\delta^{5}}{d_{K}}\right) .
\end{aligned}
$$

See (27). Thus, the error analysis has been improved from $O\left(\widehat{\delta}^{5} / d_{K}^{2}\right)$ to $O\left(\widehat{\delta}^{5} / d_{K}\right)$, which is contributed by each triangle in $T_{2}$.

Let

$$
d(P) \equiv d=\min \left\{d_{K} \mid P \notin \Delta_{K}, K=1, \ldots, N\right\} .
$$

For simplicity, we take $d_{K} \doteq d, 2 d, \ldots$, depending on how far the $\Delta_{K}$ is from the point $P$. (A somewhat more complicated argument can be based on a lower bound of a similar type for $d_{K}$.) Let $r=\widehat{\delta} / d$, which is finite for our uniform mesh subdivision scheme, and $d=$ $O\left(\widehat{\delta}_{N}\right)$. Note the indexing $\Delta_{1}, \ldots, \Delta_{N}$ does not indicate distance from $P$. But there is an arrangement of $\left\{\Delta_{K}\right\}$ where the number of triangles at a distance $R$ is proportional to $R$, with $R=d, 2 d, 3 d, \ldots$.

The number $c_{i}$ of triangles in $T_{1}$ at a distance $i \cdot d$ is proportional to $i$ for $i=1, \ldots, t_{j}$. Note that for some integer $t_{j}, t_{j} \cdot d$ is the longest possible distance from $P$ to triangles in $R_{j}$. 
Adding the error contributed by each triangle in $T_{1}$, we have

$$
\sum_{K} O\left(\frac{\widehat{\delta}_{K}^{6}}{d_{K}^{2}}\right)=\sum_{i=1}^{t_{j}} c_{i} \cdot O\left(\frac{\widehat{\delta^{6}}}{(i \cdot d)^{2}}\right)=O\left(\widehat{\delta}^{4}\right) \sum_{i=1}^{t_{j}} r^{2} \frac{i}{i^{2}}=O\left(\widehat{\delta}^{4} \log \widehat{\delta}\right) .
$$

The error contributed by each of the triangles in $T_{2}$ is $O\left(\widehat{\delta}_{K}^{5} / d_{K}\right)$. The number of triangles of this type at a distance $i \cdot d$ is a finite number, usually two or three; the proof is omitted. Therefore,

$$
\sum_{K} O\left(\frac{\widehat{\delta}_{K}^{5}}{d_{K}}\right)=\sum_{i=1}^{t_{j}} c_{j}^{\prime} \cdot O\left(\frac{\widehat{\delta}^{4}}{i \cdot d}\right)=O\left(\widehat{\delta}^{4}\right) \sum_{i=1}^{t_{j}} r \frac{1}{i}=O\left(\widehat{\delta}^{4} \log \widehat{\delta}\right),
$$

where $c_{j}^{\prime}$ is either two or three. This completes the proof that

$$
\mathcal{K}\left(I-\mathcal{P}_{N}\right) \rho(P)=O\left(\widehat{\delta}^{4} \log \widehat{\delta}\right)
$$

uniformly for $P$ a node point in the triangulation $\left\{\Delta_{K, N}\right\}$ of $S$. (This form of proof is also used in some of the remaining proofs of this paper.)

(b) To show (22), we first note that the error equation for the iterated collocation solution $\hat{\rho}_{N}$ is given by

$$
\left(2 \pi+\mathcal{K} \mathcal{P}_{N}\right)\left(\rho-\hat{\rho}_{N}\right)=-\mathcal{K}\left(I-\mathcal{P}_{N}\right) \rho
$$

The linear system associated with this is

$$
\left(2 \pi+K_{N}\right) e_{N}=-\epsilon_{N}
$$

with

$$
\begin{aligned}
& e_{N, i}=\rho\left(v_{i}\right)-\hat{\rho}_{N}\left(v_{i}\right)=\rho\left(v_{i}\right)-\rho_{N}\left(v_{i}\right), \\
& \epsilon_{N, i}=\mathcal{K}\left(I-\mathcal{P}_{N}\right) \rho\left(v_{i}\right), \quad i=1, \ldots, N_{v} .
\end{aligned}
$$

The matrix of coefficients $2 \pi+K_{N}$ is also the same as that for the linear system associated with the collocation equation (18).

As noted earlier following $(21),\left(2 \pi+\mathcal{K} \mathcal{P}_{N}\right)^{-1}$ is uniformly bounded for all sufficiently large $N$. Also, since the iterated collocation equation can be considered a Nyström method, it is a standard derivation that

$$
\left\|\left(2 \pi+K_{N}\right)^{-1}\right\| \leq\left\|\left(2 \pi+\mathcal{K} \mathcal{P}_{N}\right)^{-1}\right\|,
$$

where the matrix norm is the standard row norm. Combining these results,

$$
\left\|\left(2 \pi+K_{N}\right)^{-1}\right\| \leq c<\infty, \quad N \geq N_{0}
$$

for some sufficiently large $N_{0}$ and some $c>0$.

Using this result with (33), and using (31) to bound $\left\|\epsilon_{N}\right\|_{\infty}$, we have the desired result (22).

3.1. The single-layer integral. For the exterior problem, we need to evaluate the corresponding single-layer integrals on the right-hand side of (5). Write

$$
\int_{S} \frac{f(Q)}{|P-Q|} d S_{Q} \approx \sum_{K=1}^{N} \int_{\sigma} \frac{f\left(\tilde{m}_{K}(s, t)\right)}{\left|P-\tilde{m}_{K}(s, t)\right|}\left|D_{s} \tilde{m}_{K}(s, t) \times D_{t} \tilde{m}_{K}(s, t)\right| d \sigma,
$$

where $P$ is one of node points. Note we are including the use of the approximating surface. 
We can see that the integrand in (35) varies from singular to quite smooth. To handle this varied behavior, we use two ways to study errors. The first case is for those $\Delta_{K}$ 's that contain the point $P$, and the second case is for the remaining $\Delta_{K}$ 's.

LEMMA 3.2. Let $P$ be a node point in $\Delta_{K}$ for some $K$. Then

$$
\begin{aligned}
& \int_{\sigma} \frac{f\left(m_{K}(s, t)\right)}{\left|P-m_{K}(s, t)\right|}\left|D_{s} m_{K}(s, t) \times D_{t} m_{K}(s, t)\right| d \sigma \\
& \quad-\int_{\sigma} \frac{f_{N}\left(\widetilde{m}_{K}(s, t)\right)}{\left|P-\tilde{m}_{K}(s, t)\right|}\left|D_{s} \tilde{m}_{K}(s, t) \times D_{t} \tilde{m}_{K}(s, t)\right| d \sigma=O\left(\widehat{\delta}_{K}^{3}\right),
\end{aligned}
$$

where $\widehat{\delta}_{K}$ is the diameter of $\widehat{\Delta}_{K}$.

Proof. There are two cases. The first case is that $P$ is a vertex in some $\Delta_{K}$, and the second case is that $P$ is a midpoint of a side of $\Delta_{K}$.

Begin with the first case and, without loss of generality, assume that

$$
P=m_{K}(0,0)=\tilde{m}_{K}(0,0)=\left(p_{1}, p_{2}, p_{3}\right) .
$$

Before proving the theorem, we show that

$$
\int_{\sigma} \frac{1}{\left|P-m_{K}(s, t)\right|} d \sigma=O\left(\widehat{\delta}_{K}^{-1}\right)
$$

Compute

$$
\begin{aligned}
& \int_{\sigma} \frac{1}{\left|P-m_{K}(s, t)\right|} d \sigma \\
& \quad=\int_{\sigma} \frac{1}{\left[\left(p_{1}-x^{1}(s, t)\right)^{2}+\left(p_{2}-x^{2}(s, t)\right)^{2}+\left(p_{3}-x^{3}(s, t)\right)^{2}\right]^{1 / 2}} d \sigma \\
& \quad=\int_{\sigma}\left[\left(s x_{s}^{1}(0,0)+t x_{t}^{1}(0,0)\right)^{2}+\left(s x_{s}^{2}(0,0)+t x_{t}^{2}(0,0)\right)^{2}\right. \\
&\left.+\left(s x_{s}^{3}(0,0)+t x_{t}^{3}(0,0)\right)^{2}+O\left(\hat{\delta}_{K}^{3}\right)\right]^{-1 / 2} d \sigma .
\end{aligned}
$$

See (14) for $x^{i}$ 's. After integrating the dominant part of the above equation using polar coordinates in the $s t$-plane about $(0,0)$, we obtain

$$
\int_{\sigma} \frac{1}{\left|P-m_{K}(s, t)\right|} d \sigma=O\left(\widehat{\delta}_{K}^{-1}\right)
$$

Now, we break the error analysis into three parts.

$$
\begin{aligned}
& \int_{\sigma} \frac{f\left(m_{K}(s, t)\right)}{\left|P-m_{K}(s, t)\right|}\left|D_{s} m_{K}(s, t) \times D_{t} m_{K}(s, t)\right| d \sigma \\
& \quad-\int_{\sigma} \frac{f_{N}\left(\tilde{m}_{K}(s, t)\right)}{\left|P-\tilde{m}_{K}(s, t)\right|}\left|D_{s} \tilde{m}_{K}(s, t) \times D_{t} \tilde{m}_{K}(s, t)\right| d \sigma \\
& \quad=E_{1}+E_{2}+E_{3},
\end{aligned}
$$


with

$$
\begin{aligned}
& E_{1}=\int_{\sigma} \frac{f\left(m_{K}(s, t)\right)-f_{N}\left(\tilde{m}_{K}(s, t)\right)}{\left|P-m_{K}(s, t)\right|}\left|D_{s} m_{K}(s, t) \times D_{t} m_{K}(s, t)\right| d \sigma, \\
& E_{2}=\int_{\sigma} \frac{f_{N}\left(\tilde{m}_{K}(s, t)\right)}{\left|P-m_{K}(s, t)\right|}\left(\left|D_{s} m_{K}(s, t) \times D_{t} m_{K}(s, t)\right|\right. \\
& \left.\quad-\left|D_{s} \tilde{m}_{K}(s, t) \times D_{t} \tilde{m}_{K}(s, t)\right|\right) d \sigma, \\
& E_{3}=\int_{\sigma}\left[\frac{1}{\left|P-m_{K}(s, t)\right|}-\frac{1}{\left|P-\tilde{m}_{K}(s, t)\right|}\right] \\
& \cdot\left|D_{s} \tilde{m}_{K}(s, t) \times D_{t} \tilde{m}_{K}(s, t)\right| f_{N}\left(\tilde{m}_{K}(s, t)\right) d \sigma .
\end{aligned}
$$

In equation (36),

$$
\left|E_{1}\right| \leq O\left(\widehat{\delta}_{K}^{3}\right) \cdot O\left(\widehat{\delta}_{K}^{2}\right) \cdot \int_{\sigma} \frac{1}{\left|P-m_{K}(s, t)\right|} d \sigma \leq O\left(\widehat{\delta}_{K}^{5}\right) \cdot O\left(\widehat{\delta}_{K}^{-1}\right)=O\left(\widehat{\delta}_{K}^{4}\right)
$$

We can easily see that equation (37) has order three:

$$
\begin{gathered}
\left|E_{2}\right| \leq \max _{s, t \in \sigma}|| D_{s} m_{K}(s, t) \times D_{t} m_{K}(s, t)|-| D_{s} \widetilde{m}_{K}(s, t) \times D_{t} \widetilde{m}_{K}(s, t)|| \\
\cdot \int_{\sigma} \frac{f_{N}\left(\tilde{m}_{K}(s, t)\right)}{\left|P-m_{K}(s, t)\right|} d \sigma=O\left(\widehat{\delta}_{K}^{4}\right) \cdot O\left(\widehat{\delta}_{K}^{-1}\right)=O\left(\widehat{\delta}_{K}^{3}\right) .
\end{gathered}
$$

For $E_{3}$, expand each $x^{i}$ about $(0,0)$, and then integrate it over $\sigma$. With a very lengthy calculation, we can show that $E_{3}$ is of order three. See [8].

If $P$ is a midpoint of a side of $\Delta_{K}$, we split $\sigma$ into two triangles, $\sigma_{1}$ and $\sigma_{2}$, and we put the singular point at a vertex in each of the new triangles; see Fig. 4 for the case with $P=m_{K}\left(\rho_{4}\right)$. We apply an affine change of variables to move again to an integral over $\sigma$. Applying the first case to these two subtriangles, we again can show the error is of order three. Thus, the error contributed by the integral over $\Delta_{K}$, which contains $P$, is always of order three, whether $P$ is a vertex or a midpoint of a side of $\Delta_{K}$.

In the next lemma, we examine the errors from integrating over those triangles $\Delta_{K}$ which do not contain $P$. Then we can combine these two lemmas and give the global error for the single layer integration.

LEMMA 3.3. Let $P$ be a node point, and consider all $\Delta_{K}$ for which $P \notin \Delta_{K}$. Then

$$
\begin{aligned}
\sum_{K} \int_{\sigma} \frac{f\left(m_{K}(s, t)\right)}{\left|P-m_{K}(s, t)\right|}\left|D_{s} m_{K}(s, t) \times D_{t} m_{K}(s, t)\right| d \sigma \\
\quad-\sum_{K} \int_{\sigma} \frac{f_{N}\left(\tilde{m}_{K}(s, t)\right)}{\left|P-\tilde{m}_{K}(s, t)\right|}\left|D_{s} \tilde{m}_{K}(s, t) \times D_{t} \tilde{m}_{K}(s, t)\right| d \sigma=O\left(\widehat{\delta}_{K}^{3}\right),
\end{aligned}
$$

where $\widehat{\delta}_{K}$ is the diameter of $\Delta_{K}$.

Proof. Since $P \notin \Delta_{K}$, we can treat the function $1 /\left|P-m_{K}(s, t)\right|$ as a smooth function. All results from Lemma 3.2 and Theorems 3.3-3.7 of [8] can be applied with slight changes. Let (39) be decomposed as $E_{1}+\cdots+E 5$ where 


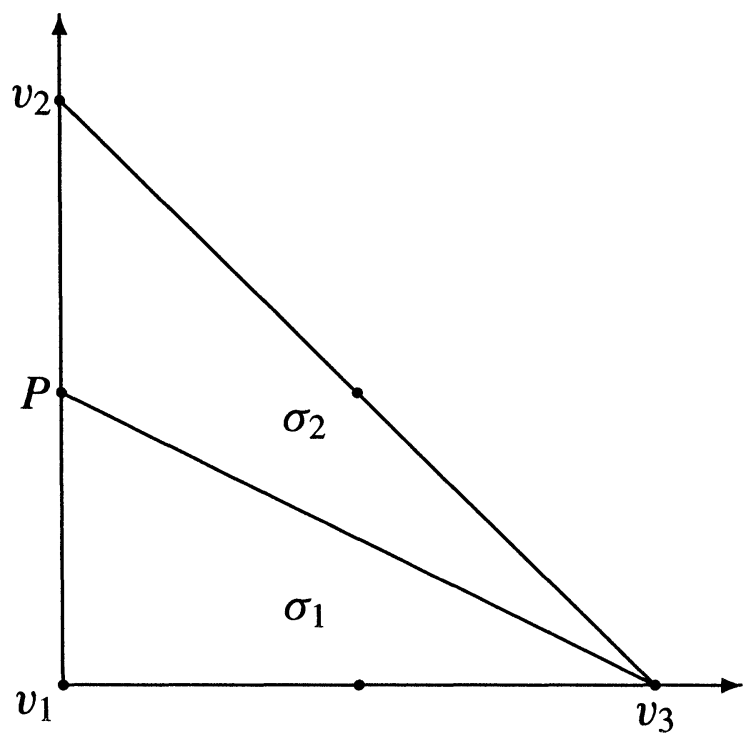

FIG. 4. Splitting triangles.

$$
\begin{aligned}
E_{1}= & \sum_{K} \int_{\sigma} \frac{f\left(m_{K}(s, t)\right)\left[\left|D_{s} m_{K} \times D_{t} m_{K}\right|-\left|D_{s} \tilde{m}_{K} \times D_{t} \tilde{m}_{K}\right|\right]}{\left|P-m_{K}(s, t)\right|} d \sigma, \\
E_{2}= & \sum_{K} \int_{\sigma} \frac{\left[f\left(m_{K}(s, t)\right)-f_{N}\left(m_{K}(s, t)\right)\right]\left|D_{s} \tilde{m}_{K} \times D_{t} \tilde{m}_{K}\right|}{\left|P-m_{K}(s, t)\right|} d \sigma \\
& -\sum_{K} \int_{\sigma} \frac{\left[f\left(m_{K}(s, t)\right)-f_{N}\left(m_{K}(s, t)\right)\right]\left|D_{s} m_{K} \times D_{t} m_{K}\right|}{\left|P-m_{K}(s, t)\right|} d \sigma, \\
E_{3}= & \sum_{K} \int_{\sigma} \frac{\left[f\left(m_{K}(s, t)\right)-f_{N}\left(m_{K}(s, t)\right)\right]\left|D_{s} m_{K} \times D_{t} m_{K}\right|}{\left|P-m_{K}(s, t)\right|} d \sigma, \\
E_{4}= & \sum_{K} \int_{\sigma}\left[\frac{f_{N}\left(m_{K}(s, t)\right)}{\left|P-m_{K}(s, t)\right|}-\frac{f_{N}\left(m_{K}(s, t)\right)}{\left|P-\tilde{m}_{K}(s, t)\right|}\right]\left|D_{s} \tilde{m}_{K} \times D_{t} \tilde{m}_{K}\right| d s d t \\
& -\sum_{K} \int_{\sigma}\left[\frac{f_{N}\left(m_{K}(s, t)\right)}{\left|P-m_{K}(s, t)\right|}-\frac{f_{N}\left(m_{K}(s, t)\right)}{\left|P-\tilde{m}_{K}(s, t)\right|}\right]\left|D_{s} m_{K} \times D_{t} m_{K}\right| d s d t, \\
E_{5}= & \sum_{K} \int_{\sigma}\left[\frac{f_{N}\left(m_{K}(s, t)\right)}{\left|P-m_{K}(s, t)\right|}-\frac{f_{N}\left(m_{K}(s, t)\right)}{\left|P-\tilde{m}_{K}(s, t)\right|}\right]\left|D_{s} m_{K} \times D_{t} m_{K}\right| d \sigma .
\end{aligned}
$$

The integrand of $E_{1}$ is $O\left(\widehat{\delta}_{K}^{6} / d_{K}^{3}\right)+O\left(\widehat{\delta}_{K}^{5} / d_{K}^{2}\right)$. Using the calculation we had in Theorem 3.1, we get that $E_{1}$ is of order three.

For $E_{2}$,

$$
\begin{aligned}
& \int_{\sigma} \frac{f\left(m_{K}(s, t)\right)-f_{N}\left(m_{K}(s, t)\right)}{\left|P-m_{K}(s, t)\right|}\left\{\left|D_{s} m_{K} \times D_{t} m_{K}\right|-\left|D_{s} \tilde{m}_{K} \times D_{t} \tilde{m}_{K}\right|\right\} d \sigma \\
& =\sum_{K} O\left(\widehat{\delta}^{3}\right) \cdot O\left(\widehat{\delta}^{4}\right) \cdot \int_{\sigma} \frac{1}{\left|P-m_{K}(s, t)\right|} d \sigma=O\left(\widehat{\delta}^{7}\right) \cdot \frac{1}{d_{K}}
\end{aligned}
$$


for every $K$ where $P \notin \Delta_{K}$. Adding errors from each triangle, we have that $E_{2}$ is $O\left(\widehat{\delta}^{5}\right)$, as we discussed in computing $E_{1}$.

For $E_{3}$, we have that the error from

$$
\int_{\sigma} \frac{f\left(m_{K}(s, t)\right)-f_{N}\left(m_{K}(s, t)\right)}{\left|P-m_{K}(s, t)\right|}\left|D_{s} m_{K} \times D_{t} m_{K}\right| d \sigma
$$

is $O\left(\widehat{\delta}^{6} / d_{K}\right)$ for every triangle. Again, following the argument in Theorem 3.1, $E_{3}$ is $O\left(\widehat{\delta}^{4}\right)$.

Analyzing $E_{4}$, we have

$$
\frac{1}{\left|P-m_{K}(s, t)\right|}-\frac{1}{\left|P-\tilde{m}_{K}(s, t)\right|}=O\left(\frac{\widehat{\delta}_{K}^{3}}{d_{K}^{2}}\right),
$$

and

$$
\begin{aligned}
\int_{\sigma} & {\left[\frac{1}{\left|P-m_{K}(s, t)\right|}-\frac{1}{\left|P-\tilde{m}_{K}(s, t)\right|}\right] f_{N}\left(m_{K}(s, t)\right)\left|D_{s} m_{K} \times D_{t} m_{K}\right| d \sigma } \\
& \left.-\int_{\sigma}\left[\frac{1}{\left|P-m_{K}(s, t)\right|}-\frac{1}{\left|P-\widetilde{m}_{K}(s, t)\right|}\right] f_{N}\left(m_{K}(s, t)\right)\left|D_{s} \tilde{m}_{K} \times D_{t} \tilde{m}_{K}\right|\right) d s d t \\
& =O\left(\frac{\widehat{\delta}_{K}^{7}}{d_{K}^{2}}\right)
\end{aligned}
$$

for each $\Delta_{K}$. After adding up errors, $E_{4}=O\left(\widehat{\delta}^{5} \ln \widehat{\delta}\right)$.

For $E_{5}$, each triangle give us an error of $O\left(\widehat{\delta}_{K}^{5} / d_{K}^{2}\right)$. When adding errors together, cancellation happens at every symmetric pair of triangles and errors become $O\left(\widehat{\delta}_{K}^{6} / d_{K}^{3}\right)$. Thus, as we discussed in computing $E_{1}, E_{5}$ is $O\left(\widehat{\delta}^{3}\right)$. After going through $E_{1}-E_{5}$, the global error for the single-layer integral, in which $P-m_{K}(s, t)$ is nonzero for every $K$, is $O\left(\widehat{\delta}^{3}\right)$. This result is uniform as $P$ ranges over the node points of the triangulation.

Combining the above lemma, we get the following result, which gives the total error for evaluating the single-layer integral at any node point. We use this later to assess the effect on $\rho_{N}$ of using an approximation to the single layer.

THEOREM 3.4. Let $S$ be a piecewise smooth surface and let $P$ be a node point on $S$. Assume the unknown function $f \in C^{4}\left(S_{i}\right) \cap C(S), i=1, \ldots, J$. Then

$$
\int_{S} \frac{f(Q)}{|P-Q|} d S_{Q}-\sum_{K=1}^{N} \int_{\sigma} \frac{f\left(\tilde{m}_{K}(s, t)\right)}{\left|P-\tilde{m}_{K}(s, t)\right|}\left|D_{s} \tilde{m}_{K}(s, t) \times D_{t} \tilde{m}_{K}(s, t)\right| d \sigma=O\left(\widehat{\delta}^{3}\right) .
$$

Proof. Combine Lemmas 3.2 and 3.3.

3.2. Using the approximate surface. When using the approximate surface $\widetilde{S}_{N}$, the linear system for (2) for the Dirichlet problem becomes

$$
\begin{aligned}
& 2 \pi \tilde{\rho}_{N}\left(v_{i}\right)+\left[2 \pi-\Omega\left(v_{i}\right)\right] \tilde{\rho}_{N}\left(v_{i}\right) \\
& \quad+\sum_{K=1}^{N} \sum_{j=1}^{6} \tilde{\rho}_{N}\left(v_{j, K}\right) \int_{\sigma} l_{j, K}(s, t) \frac{\left(D_{s} \tilde{m}_{K}(s, t) \times D_{t} \tilde{m}_{K}(s, t)\right) \cdot\left(v_{i}-\tilde{m}_{K}(s, t)\right)}{\left|v_{i}-\tilde{m}_{K}(s, t)\right|^{3}} d \sigma \\
& \quad=f\left(v_{i}\right), \quad i=1, \ldots N_{v} .
\end{aligned}
$$

For a smooth surface $S$, we would expect to use $\Omega_{N}(P)=\Omega(P)=2 \pi$, thus simplifying the above system. However, for the piecewise smooth surfaces considered in $\S 5$, we need to 
consider an approximation to $\Omega(P)$, and from the numerical examples in $\S 4$, it is also useful to consider approximations of $\Omega(P)$ for $S$ a smooth surface.

Using the identity

$$
\Omega(P)=\int_{S} \frac{\partial}{\partial \nu_{Q}}\left[\frac{1}{|P-Q|}\right] d S_{Q}, \quad P \in S,
$$

we define

$$
\Omega_{N}(P)=\sum_{K=1}^{N} \sum_{j=1}^{6} \int_{\sigma} l_{j, K}(s, t) \frac{\left(D_{s} \tilde{m}_{K} \times D_{t} \tilde{m}_{K}\right) \cdot\left(P-\tilde{m}_{K}(s, t)\right)}{\left|P-\tilde{m}_{K}(s, t)\right|^{3}} d \sigma .
$$

Later, in Theorem 5.2 of $\S 5$, we show that

$$
\max _{1 \leq i \leq N_{v}}\left|\Omega\left(v_{i}\right)-\Omega_{N}\left(v_{i}\right)\right|=O\left(\widehat{\delta}_{N}^{2}\right) .
$$

Empirically for a smooth surface $S$, as shown in $\S 4$, it appears the approximation error is actually $O\left(\widehat{\delta}_{N}^{3}\right)$, although we have not been able to prove this.

The linear system (41) is denoted here by

$$
\left(2 \pi+\widetilde{K}_{N}\right) \widetilde{\rho}_{N}=g_{N},
$$

with

$$
\tilde{\rho}_{N, i} \equiv \tilde{\rho}_{N}\left(v_{i}\right), \quad g_{N, i} \equiv ; f_{N}\left(v_{i}\right), \quad i=1, \ldots, N_{v} .
$$

When solving the integral equation (5) for the exterior Neumann problem, we also approximate the right-hand side, now a single-layer integral, using (35). In the above framework, and consistent with earlier notation, we write

$$
\tilde{g}_{N, i}=\sum_{K=1}^{N} \sum_{j=1}^{6} \int_{\sigma} \frac{f\left(\tilde{m}_{K}(s, t)\right)\left|D_{s} \tilde{m}_{K} \times D_{t} \tilde{m}_{K}\right|}{\left|v_{i}-\tilde{m}_{K}(s, t)\right|} d \sigma
$$

for $i=1, \ldots, N_{v}$.

For convergence when using the approximate surface $\widetilde{S}_{N}$, we have the following theorem. In $\S 4$, we give experimental results which suggest that the convergence results given below can be improved.

THEOREM 3.5. Consider the integral equations (2) and (5) with solution $\rho$. Let $S$ be a smooth surface in $\mathbf{R}^{3}$ and assume the unknown function $\rho \in C^{4}(S)$. Then

$$
\max _{1 \leq i \leq N_{v}}\left|\rho\left(v_{i}\right)-\widetilde{\rho}_{N}\left(v_{i}\right)\right|=O\left(\widehat{\delta}_{N}^{2}\right) .
$$

Proof. We use a perturbation analysis, based on regarding the system (45) as a perturbation of the corresponding system

$$
\left(2 \pi I+K_{N}\right) \rho_{N}=g_{N},
$$

for the projection method analyzed in Theorem 3.1 which used the exact surface $S$. As shown earlier in (34), $\left(2 \pi+K_{N}\right)^{-1}$ is uniformly bounded for all sufficiently large $N$.

The present analysis uses the result

$$
\left\|K_{N}-\widetilde{K}_{N}\right\|=O\left(\widehat{\delta}_{N}^{2}\right)
$$


with the matrix row norm. The proof of this is essentially the same as that for (44), and thus we defer the proof of (60) to Theorem 5.2. Using (49) and the invertibility of $2 \pi+K_{N}$ with the uniform boundness of $\left(2 \pi+K_{N}\right)^{-1}$, for all sufficiently large $N$, we have by standard arguments that the same is true for the inverse of $2 \pi+\widetilde{K}_{N}$ :

$$
\left\|\left(2 \pi+\widetilde{K}_{N}\right)^{-1}\right\| \leq c<\infty, \quad N \geq N_{0}
$$

for some $N_{0}$ and some $c>0$.

By straightforward manipulation of (45) and (48), we have

$$
\rho_{N}-\widetilde{\rho}_{N}=\left(2 \pi+\widetilde{K}_{N}\right)^{-1}\left[\widetilde{K}_{N}-K_{N}\right] g_{N}+\left(2 \pi+\widetilde{K}_{N}\right)^{-1}\left[g_{N}-\widetilde{g}_{N}\right] .
$$

The first term on the right-hand side is $O\left(\widehat{\delta}_{N}^{2}\right)$, from (49). The second term is either zero or $O\left(\widehat{\delta}_{N}^{3}\right)$, from Theorem 3.4. When considered with Theorem 3.1, this shows the result (47).

4. Numerical examples: Smooth surface case. The collocation method of $\S 3$, with the use of the quadratic isoparametric interpolation of the surface $S$, was implemented with a package of programs which work for a wide variety of smooth and piecewise smooth surfaces. This package was first described in [2], [3]; it has since been updated and improved in several ways. (Eventually the package will be made available publicly, with an accompanying user's manual.)

There are two crucial aspects of the practical implementation that were not discussed in $\S 3$ : the calculation of the collocation integrals and the solution of the large linear systems that often arise from the discretization. The iterative solution of such linear systems by two-grid methods is discussed in [6], [7]; thus we restrict our attention here to the numerical integration of the collocation integrals.

After much experimentation with other approaches, we have currently settled on the following scheme for the numerical integration. We find that the numerical integration of the collocation integrals is by far the most time-consuming part in solving the boundary integral equation. One must have integrals that are sufficiently accurate to match the accuracy of the "pure" collocation solution $\rho_{N}$. But it is very wasteful of computing time to calculate these integrals with more accuracy than is needed.

The collocation integrals in the matrix of coefficients of (41) are given by

$$
\int_{\sigma} \kappa\left(v_{i}, \tilde{m}_{k}(s, t)\right) l_{j}(s, t)\left|D_{s} \tilde{m}_{K} \times D_{t} \tilde{m}_{k}\right| d \sigma
$$

In this, $i=1, \ldots, N_{v}, j=1, \ldots, 6$, and $K=1, \ldots, N ; \kappa(P, Q)$ denotes the kernel function for the double layer integral operator. For the exterior Neumann problem, we also need to evaluate the corresponding single-layer integrals

$$
\int_{\sigma} \frac{f\left(\tilde{m}_{K}(s, t)\right)}{\left|v_{i}-\tilde{m}_{K}(s, t)\right|}\left|D_{s} \tilde{m}_{K} \times D_{t} \tilde{m}_{K}\right| d \sigma .
$$

Recall from $\S 2$ that $\tilde{m}_{K}: \sigma \longrightarrow \widetilde{\Delta}_{K}$ is a one-to-one and onto parametrization of the triangle approximating $\Delta_{K}$. We consider two cases in evaluating (52), depending on whether $v_{i}$ is inside or outside of $\Delta_{K}$.

If $v_{i} \in \Delta_{K}$, then $\kappa\left(v_{i}, Q\right)$ is singular. We use a change of variable based on [9]. This was introduced in [3, p. 40], where we noted that it removed all singular behavior in both the double-layer integrals (52) and the corresponding single-layer integrals. Subsequently, we discovered that the change of variables is equivalent to that introduced in [11]. Others who 
have since made use of this transformation include [13] and [21]. The latter paper carries out a detailed analysis of the method and an extension of the transformation to other singular integration problems arising in solving boundary integral equations.

Assuming the collocation node $v_{i}=m_{K}(0,0)$, introduce the change of variables

$$
s=(1-y) x, \quad t=y x, \quad 0 \leq x, y \leq 1 .
$$

With this, the new integrands in (52) and (53) will be well behaved. For $\Delta_{K}$ a surface with $C^{m}$ differentiability, $m \geq 3$, the transformed integrand for (52) will be $C^{m-2}$ times differentiable; if the density $f(Q)$ is $m$-times differentiable on $\Delta_{K}$, the transformed integrand for (53) will be $C^{m-1}$ times differentiable. We then evaluate the transformed integral using a product Gaussian quadrature formula, with $N_{g}$ nodes in both the $x$ and $y$ coordinates (thus using $N_{g}^{2}$ integration nodes). If $v_{i}=m_{K}(0,1)$ or $m_{K}(1,0)$, then we use an affine transformation to convert back to the case just discussed. If $v_{i}=m_{K}\left(q_{j}\right)$ with $j=4,5$, or 6 , then we divide $\sigma$ into two parts and treat the integral over each part as described above. As an example, suppose $v_{i}=m_{K}(0, .5)$. See Fig. 4 for the appropriate subdivision of $\sigma$, for which we use an affine transformation to map each subtriangle onto $\sigma$ in such a way that the singular point occurs at $(0,0)$.

The above change of variables is used to remove the singularity in the integration over each triangle. For cases of $N=512$ faces, we have found $N_{g}=10$ to be very sufficient to preserve the accuracy of the collocation solution; smaller values of $N_{g}$ are sufficient for smaller values of $N$. Note that the number of integrals (52) with $v_{i} \in \Delta_{K}$, for some $i$ and $K$, is of order $N_{v}$, whereas the total number of integrals to be computed is of order $N_{v}^{2}$. Thus when considering operation counts, the singular integrals are the less important of the integrals (52) to be considered.

For $v_{i} \notin \Delta_{K}$, the integrand in (52) is analytic, but it is increasingly peaked as the distance between $v_{i}$ and $\Delta_{K}$ decreases. A method to evaluate integrals such as (52) and (53) over $\sigma$ is based on (17), the quadrature rule T2:5-1 of [22]. Let an integer parameter $N_{d} \geq 0$ be given. If $v_{i} \notin \Delta_{K}$ and

$$
\operatorname{dist}\left(v_{i}, \Delta_{K}\right) \leq \delta_{N}
$$

where $\delta_{N}$ is the mesh size of $\left\{\Delta_{K}\right\}$ as defined in (9), then integrate (52) using (17) with $N_{d}$ levels of subdivision of $\sigma$ (thus dividing $\sigma$ into $4^{N_{d}}$ subtriangles, with (17) applied to the integral over each of the corresponding subintegrals). If $v_{i} \notin \Delta_{K}$ and

$$
\delta_{N}<\operatorname{dist}\left(v_{i}, \Delta_{K}\right) \leq 2 \delta_{N},
$$

then integrate (52) using (17) with $\max \left\{N_{d}-1,0\right\}$ levels of subdivision of $\sigma$. If $v_{i} \notin \Delta_{K}$ and

$$
2 \delta_{N}<\operatorname{dist}\left(v_{i}, \Delta_{K}\right) \leq 3 \delta_{N}
$$

then integrate (52) using (17) with $\max \left\{N_{d}-2,0\right\}$ levels of subdivision of $\sigma$. Continue with this in the obvious way.

We have found that as $N$ is increased to $4 N$, then raising $N_{d}$ to $N_{d}+1$ is sufficient to integrate (52) and (53) with the needed accuracy. For all of our examples, for both smooth and piecewise smooth surfaces, the largest value of $N_{d}$ that we have needed to use has been $N_{d}=2$. We have used larger values of $N_{d}$ in our experiments to check the accuracy when using the lower values of $N_{d}$. When $v_{i} \notin \Delta_{K}$, other methods have been tried for evaluating (52) and (53); for example, a method with automatic error control was described in [3] and [4]. But the method described here has proven to be the most efficient. Nonetheless, the integrations of (52) and (53) are still the most expensive parts of our computation, far exceeding the cost of solving the linear system (23) for the discretized boundary integral equation. 


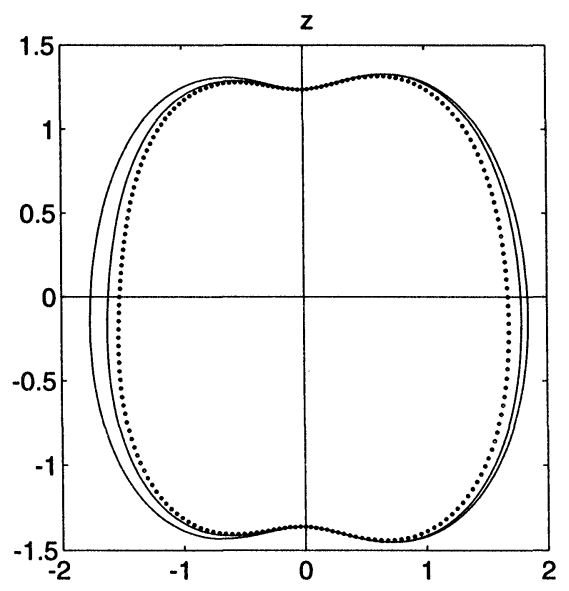

solid curve: $\phi=0, \quad$ dash curve: $\phi=\pi / 4, \quad$ dot curve: $\phi=\pi / 2$

FIG. 5. Cross-sections of "squash" surface.

4.1. The surfaces. Two smooth surfaces were used in our experiments. Surface \#1 (denoted by $S \# 1$ ) was the ellipsoid

$$
\left(\frac{x}{a}\right)^{2}+\left(\frac{y}{b}\right)^{2}+\left(\frac{z}{c}\right)^{2}=1 .
$$

In Tables 1-4 given below for this ellipsoid, $(a, b, c)=(2,2.5,3)$.

The ellipsoid is convex and symmetric. For that reason, we also devised and used a surface which is not symmetric and which is slightly nonconvex. Surface \#2 (S\#2) is defined by

$$
(x, y, z)=\rho(\xi, \eta, \zeta)(A \xi, B \eta, C \zeta), \quad \xi^{2}+\eta^{2}+\zeta^{2}=1,
$$

with

$$
\rho(\xi, \eta, \zeta)=1-\left[(\xi-.1)^{2}+2(\eta-.1)^{2}-3(\zeta-.1)^{2}\right] / \alpha
$$

and $A, B, C>0, \alpha \geq 5$. The case we use here is $\alpha=10$ and $(A, B, C)=(2,2,1)$. Figure 5 gives the cross-sections of $S \# 2$ when intersecting $S$ with vertical planes containing the $z$-axis, intersecting at angles of $\phi=0, \pi / 4, \pi / 2$ with respect to the positive $x$-axis. Experiments were done with other choices of $\alpha$ and $(A, B, C)$, corresponding to surfaces with a more pronounced lack of symmetry and convexity. But in order to obtain error results with some regularity in asymptotic behavior, we chose the parameters given above, giving the surface illustrated in Fig. 5.

4.2. The solid angle. At all points $P \in S$, the solid angle $\Omega(P)=2 \pi$. In Table 1 , we give the approximate values of the solid angle for $S \# 1$ as computed using $\Omega_{N}(P)$ in (43). The points $P$ at which these are given are

$$
\begin{array}{lll}
v_{1}=(0,0,3), & v_{2}=(2,0,0), & v_{3}=(0,2.5,0), \\
v_{7}=(\sqrt{2}, \sqrt{4.5}, 0), & v_{8}=(\sqrt{2}, \sqrt{3.125}, 0), & v_{9}=(0, \sqrt{3.125}, \sqrt{4.5})
\end{array}
$$

The subscripts refer to the indexing of node points used in our triangulation package. The empirical rate of convergence is approximately $O\left(\widehat{\delta}_{N}^{3}\right)$. The integration parameters used were 
TABLE 1

Solid angle approximations on $\$$ \#1 at selected $v_{i}$.

\begin{tabular}{ccccccc}
\hline$i$ & $E 1$ & $E 2$ & $E 3$ & $E 1 / E 2$ & $E 2 / E 3$ & $E 3 / E 4$ \\
\hline 1 & $1.52 \mathrm{E}-1$ & $2.01 \mathrm{E}-2$ & $2.54 \mathrm{E}-3$ & 6.58 & 7.58 & 7.89 \\
2 & $8.26 \mathrm{E}-2$ & $1.09 \mathrm{E}-2$ & $1.38 \mathrm{E}-3$ & 6.64 & 7.58 & 7.89 \\
3 & $1.17 \mathrm{E}-1$ & $1.55 \mathrm{E}-2$ & $1.96 \mathrm{E}-3$ & 6.62 & 7.58 & 7.89 \\
7 & $1.73 \mathrm{E}-1$ & $2.35 \mathrm{E}-2$ & $3.01 \mathrm{E}-3$ & 8.13 & 7.34 & 7.82 \\
8 & $1.47 \mathrm{E}-1$ & $2.00 \mathrm{E}-2$ & $2.56 \mathrm{E}-3$ & 7.55 & 7.36 & 7.82 \\
9 & $2.34 \mathrm{E}-1$ & $3.19 \mathrm{E}-2$ & $4.09 \mathrm{E}-3$ & 8.29 & 7.32 & 7.81 \\
\hline
\end{tabular}

TABLE 2

Maximum errors on ellipsoid.

\begin{tabular}{rcccc}
\hline & $\left\|u_{1}-u_{1 N}\right\|_{\infty}$ & Ratio & $\left\|u_{2}-u_{2 N}\right\|_{\infty}$ & Ratio \\
\hline 8 & $1.93 \mathrm{E}-2$ & & $1.92 \mathrm{E}-2$ & \\
32 & $1.44 \mathrm{E}-3$ & 13.4 & $2.85 \mathrm{E}-3$ & 6.7 \\
128 & $9.68 \mathrm{E}-5$ & 14.9 & $2.54 \mathrm{E}-4$ & 11.2 \\
512 & $6.09 \mathrm{E}-6$ & 15.9 & $1.63 \mathrm{E}-5$ & 15.6 \\
\hline
\end{tabular}

TABLE 3

Maximum errors on surface $\mathrm{S \# 2.}$

\begin{tabular}{rcrcr}
\hline & $\left\|u_{1}-u_{1 N}\right\|_{\infty}$ & Ratio & $\left\|u_{2}-u_{2 N}\right\|_{\infty}$ & Ratio \\
\hline 8 & $7.26 \mathrm{E}-2$ & & $5.49 \mathrm{E}-2$ \\
32 & $5.40 \mathrm{E}-3$ & 13.4 & $4.85 \mathrm{E}-3$ & 11.3 \\
128 & $8.70 \mathrm{E}-4$ & 6.2 & $1.35 \mathrm{E}-3$ & 3.6 \\
512 & $1.11 \mathrm{E}-4$ & 7.9 & $1.98 \mathrm{E}-4$ & 6.8 \\
\hline
\end{tabular}

$N_{g}=10$ and $N_{d}=2$. The columns $E 1, E 2, E 3$, and $E 4$ denote the errors for $N=8,32$, 128,512 , respectively. (Note that for a given $N$, the number of nodes on $S$ is $N_{v}=2(N+1)$.) Similar results for the approximate solid angle are true for $S \# 2$.

4.3. Solution of the exterior Neumann problem. The problem (3) was solved with the normal derivative $f$ so chosen that the true solution is known. The two cases used here are

$$
u_{1}(P)=\frac{1}{r}, \quad u_{2}(P)=\frac{1}{r} e^{x / r^{2}} \cos \left(z / r^{2}\right),
$$

with $P=(x, y, z)$ and $r=|P|$. In this case, $\rho=u$; and we use $u$ and $u_{N}$ in our discussion. Tables 2 and 3 contain the maximum error at the node points for solving boundary integral equation (5) for $S \# 1$ and $S \# 2$, respectively. The integration parameter $N_{g}=10$; for $N_{d}$, we used $0,1,2,2$ for the cases $N=8,32,128$, and 512, respectively, for both $S \# 1$ and $S \# 2$.

The results in Table 2 for $S \# 1$ are consistent with an asymptotic rate for the error of $O\left(\widehat{\delta}_{N}^{4}\right)$ or $O\left(\widehat{\delta}_{N}^{4} \log \widehat{\delta}_{N}\right)$, in agreement with the theoretical result in Theorem 3.1 for the collocation method with the exact surface. In the case of $S \# 2$ in Table 3, the asymptotic pattern for the maximum error appears to be $O\left(\widehat{\delta}_{N}^{3}\right)$; to check in more detail whether the error is truly $O\left(\widehat{\delta}_{N}^{3}\right)$ refer to Table 4, which gives the errors at a representative sampling of the 18 nodes used in the coarsest triangulation of $S$ (for $N=8$ ), along with the ratios by which these errors decrease. The columns $E 1, E 2, E 3$, and $E 4$ denote the errors for the parameter $N=8,32,128$, and 512 , respectively. When looking at the individual errors, there is a pattern of an $O\left(\widehat{\delta}_{N}^{4}\right)$ rate of convergence at a large number of the points; we conjecture that with larger values of $N$, an asymptotic error of $O\left(\widehat{\delta}_{N}^{4}\right)$ would emerge for the maximum error.

Since these are smooth surfaces, why not use the true value of $\Omega\left(v_{i}\right)=2 \pi$, rather than incorporating the approximation (43) into the discretization of (5)? Table 5 gives the values 
TABLE 4

Errors at representative $v_{i}$ on $S \# 2$ for $u=u_{1}$.

\begin{tabular}{rcccccc}
\hline$N$ & $E 2$ & $E 3$ & $E 4$ & $E 1 / E 2$ & $E 2 / E 3$ & $E 3 / E 4$ \\
\hline 1 & $-5.26 \mathrm{E}-3$ & $-4.77 \mathrm{E}-4$ & $-3.14 \mathrm{E}-5$ & 8.4 & 11.0 & 15.2 \\
2 & $-4.25 \mathrm{E}-3$ & $-2.81 \mathrm{E}-4$ & $-1.78 \mathrm{E}-5$ & 11.3 & 15.1 & 15.8 \\
5 & $-5.41 \mathrm{E}-3$ & $-3.28 \mathrm{E}-4$ & $-2.24 \mathrm{E}-5$ & 13.4 & 16.5 & 14.6 \\
7 & $-3.09 \mathrm{E}-3$ & $-8.05 \mathrm{E}-5$ & $1.30 \mathrm{E}-5$ & 16.9 & 38.4 & -6.2 \\
8 & $-4.58 \mathrm{E}-3$ & $-3.80 \mathrm{E}-4$ & $-2.62 \mathrm{E}-5$ & 10.9 & 12.0 & 14.5 \\
12 & $-5.40 \mathrm{E}-3$ & $-4.63 \mathrm{E}-4$ & $-3.35 \mathrm{E}-5$ & 9.6 & 11.7 & 13.8 \\
13 & $-3.16 \mathrm{E}-3$ & $-1.52 \mathrm{E}-4$ & $2.84 \mathrm{E}-6$ & 16.8 & 20.8 & -53.3 \\
15 & $-2.52 \mathrm{E}-3$ & $-7.91 \mathrm{E}-5$ & $9.18 \mathrm{E}-6$ & 18.2 & 31.9 & -8.6 \\
18 & $-2.51 \mathrm{E}-3$ & $-1.51 \mathrm{E}-4$ & $-3.04 \mathrm{E}-6$ & 17.7 & 16.6 & 49.8 \\
\hline
\end{tabular}

TABLE 5

Errors for $u=u_{1}$ on the ellipsoid S\#1 with use of the exact solid angle $\Omega=2 \pi$.

\begin{tabular}{rcc}
\hline$N$ & $\left\|u_{1}-u_{1 N}\right\|_{\infty}$ & Ratio \\
\hline 8 & $9.75 \mathrm{E}-2$ & \\
32 & $1.35 \mathrm{E}-2$ & 7.24 \\
128 & $3.26 \mathrm{E}-3$ & 4.13 \\
512 & $4.37 \mathrm{E}-4$ & 7.45 \\
\hline
\end{tabular}

of the maximum error at the node points $\left\{v_{i}\right\}$ with $u=u_{1}$ on $S \# 1$, with $\Omega\left(v_{i}\right)=2 \pi$ at all node points. Note that now the error is $O\left(\widehat{\delta}_{N}^{3}\right)$, which is worse than the convergence rate of $O\left(\widehat{\delta}_{N}^{4} \log \widehat{\delta}_{N}\right)$ predicted by Theorem 3.1 for the solution $u_{N}$.

The use of the approximation (43) forces a favorable cancellation to occur when forming the discretized linear system (41). Another way of looking at what is happening is the following. The matrix of coefficients (41) is forced to have $4 \pi$ as an eigenvalue, with the eigenvector being the vector with all components equal to 1 . This makes the discretized system exactly like the original integral equation (5), in which the function $u(P) \equiv 1$ is an eigenfunction of the left-hand side of (5), with the eigenvalue being $4 \pi$.

It is clearly preferable to use the approximate solid angle rather than the exact one. The cost of using the approximation (43) is minimal, since all quantities used have been calculated in setting up the linear system (41).

4.4. The interior Dirichlet problem. We solve the integral equation (2) for the interior Dirichlet problem, using the same procedures described above for the exterior Neumann problem. To complete the solution process, we must then calculate numerically the integral (1). Letting $\tilde{\rho}_{N}$ denote the approximate density function thus obtained, we must evaluate

$$
u_{N}(A)=\int_{S} \tilde{\rho}_{N}(Q) \frac{\partial}{\partial \nu_{Q}}\left[\frac{1}{|A-Q|}\right] d S_{Q}, \quad A \in D .
$$

From [8], the rate of convergence will be $O\left(\widehat{\delta}_{N}^{4}\right)$ when the quadrature is based on standard symmetric numerical integration rules over the unit simplex with a sufficiently high degree of precision, e.g., the rules (16) and (17).

Expand the integral in (54) as

$$
\sum_{k=1}^{n} \int_{\Delta_{K}} \tilde{\rho}_{N}(Q) \frac{\partial}{\partial v_{Q}}\left[\frac{1}{|A-Q|}\right] d S_{Q}
$$

The triangulation $\left\{\Delta_{K}\right\}$ used here need not be the same as the one used in obtaining $\tilde{\rho}_{N}$, but the two triangulations should be compatible in the sense that one is a refinement of the other. 
For those triangles $\Delta_{K}$ which are close to the field point $A$, the integration should be done with more accuracy than for those triangles which are relatively far from the field point.

It has been our experience that the density function $\tilde{\rho}_{N}$ can be relatively inaccurate and quite acceptable accuracy in the solution $u_{N}(A)$ can still be obtained. The accuracy in the solution $u_{N}$ depends much more on the accuracy of the numerical integration of (55) than on having high accuracy in $\tilde{\rho}_{N}$. This should not be especially surprising since it is well known that integration is a "smoothing" operation and the effect of errors in the integrand, including $\tilde{\rho}_{N}$, are reduced. Extended examples to illustrate this are given in the technical report [4], and we omit them here for reasons of space.

5. Collocation on piecewise smooth boundaries. As in $\S 3$, we first analyze the collocation method $\left(2 \pi+\mathcal{P}_{N} \mathcal{K}\right) \rho_{N}=\mathcal{P}_{N} \mathcal{S} f$ for (5) by assuming the exact representation of the surface is used in all integrations; following that, we analyze the effect of using a quadratic interpolatory representation of the surface. For polyhedral boundaries, there is no need to approximate the boundary, and these are the cases analyzed in [10] and [17].

As in [2], we use a stability analysis based on Wendland [23]; then as in $\S 3$, we analyze the discretization error for the iterated collocation solution

$$
\hat{\rho}_{N}=\frac{1}{2 \pi}\left(g-\mathcal{K} \rho_{N}\right) .
$$

In [23], a piecewise constant collocation method is defined and analyzed. The proofs given there generalize easily to our collocation method based on quadratic isoparametric interpolation. In his paper, Wendland makes several assumptions about the piecewise smooth surface $S$, in addition to those described in $\S 2$. Assumption V3 of his paper states that at all points of $S$, either the inner or the outer tangent cone must be convex; assumption V4 states that all edges of $S$ must be piecewise continuous and must not contain any cusps. Within this setting, it is straightforward to prove the following theorem.

THEOREM 5.1. Let $S$ satisfy the assumptions given above and earlier in $\S 2$; and let $S$ also satisfy the assumptions V3 and V4 of [23], as discussed above preceding the theorem. Moreover, assume

$$
\frac{5}{3} \sup _{P \in S}|2 \pi-\Omega(P)|<2 \pi .
$$

Let $\mathcal{P}_{N}$ denote the interpolatory projection of (12), based on quadratic isoparametric interpolation over the triangulation $\left\{\Delta_{K} \mid K=1, \ldots, N\right\}$. Then for all sufficiently large $N$, say $N \geq N_{0}$, and for some $c<\infty$,

$$
\left\|\left(2 \pi+\mathcal{P}_{N} \mathcal{K}\right)^{-1}\right\| \leq c, \quad N \geq N_{0} .
$$

Moreover, this implies that

$$
\left\|\left(2 \pi+\mathcal{K} \mathcal{P}_{N}\right)^{-1}\right\| \leq c, \quad N \geq N_{0}
$$

for the error

$$
\left\|\rho-\rho_{N}\right\| \leq O\left(\widehat{\delta}_{N}^{3}\right)
$$

Proof. We refer to the derivation in [23]. Essentially, the problem of analyzing $\left(2 \pi+\mathcal{P}_{N} \mathcal{K}\right) \rho_{N}=\mathcal{P}_{N} g$ is divided into two parts. Begin by decomposing the surface $S$ into two subdomains based on distance to an edge or vertex of $S$. Let $T$ denote the union of all edges and vertices of the surface $S$. For a given $\epsilon>0$, let

$$
S_{1}=\{P \in S \mid \operatorname{dist}(P, T) \leq \epsilon\}
$$


and let $S_{2}$ be the closure of $S-S_{1}$. Consider spaces $C\left(S_{i}\right), i=1,2$, and define integral operators $\mathcal{K}_{i j}: C\left(S_{i}\right) \rightarrow C\left(S_{j}\right)$ by

$$
\left(\mathcal{K}_{i j} \rho\right)(P)=\int_{S_{j}} \rho(Q) \frac{\partial}{\partial \nu_{Q}}\left[\frac{1}{|P-Q|}\right] d S_{Q}+[2 \pi-\Omega(P)] \rho(P), P \in S_{i}, \rho \in C\left(S_{j}\right) .
$$

The final term $[2 \pi-\Omega(P)] \rho(P)$ needs to be included only when $i=j=1$. For $(i, j) \neq$ $(1,1)$, the operators $\mathcal{K}_{i j}$ are compact.

Define $\mathcal{X} \equiv C\left(S_{i}\right) \oplus C\left(S_{j}\right)$. Then the original boundary integral equation (5) for the exterior Neumann problem, $(2 \pi+\mathcal{K}) \rho=\mathcal{S} f$, and the collocation equation for its solution, $\left(2 \pi+\mathcal{P}_{N} \mathcal{K}\right) \rho_{N}=\mathcal{P}_{N} \mathcal{S} f$, can be reformulated, respectively, as

$$
\begin{aligned}
& {\left[\begin{array}{cc}
2 \pi+\mathcal{K}_{11} & \mathcal{K}_{12} \\
\mathcal{K}_{21} & 2 \pi+\mathcal{K}_{22}
\end{array}\right]\left[\begin{array}{l}
\rho_{1} \\
\rho_{2}
\end{array}\right]=\left[\begin{array}{l}
g_{1} \\
g_{2}
\end{array}\right],} \\
& {\left[\begin{array}{cc}
2 \pi+\mathcal{P}_{1 N} \mathcal{K}_{11} & \mathcal{P}_{1 N} \mathcal{K}_{12} \\
\mathcal{P}_{2 N} \mathcal{K}_{21} & 2 \pi+\mathcal{P}_{2 N} \mathcal{K}_{22}
\end{array}\right]\left[\begin{array}{l}
\rho_{1 N} \\
\rho_{2 N}
\end{array}\right]=\left[\begin{array}{l}
\left(\mathcal{P}_{N} g\right)_{1} \\
\left(\mathcal{P}_{N} g\right)_{2}
\end{array}\right] .}
\end{aligned}
$$

We assume that the interpolation operator $\mathcal{P}_{N}$ is defined so that $\mathcal{P}_{N} \rho \mid S_{i}$ depends on $\rho$ at only the node points within $S_{i}$. Then we can define $\mathcal{P}_{i}: C\left(S_{i}\right) \rightarrow C\left(S_{i}\right)$ by

$$
\mathcal{P}_{i N} \rho=\mathcal{P}_{N} \rho \mid S_{i}, \quad \rho \in C\left(S_{i}\right), \quad i=1,2 .
$$

Using the methods of [23], it is straightforward to show that if $\epsilon$ is chosen sufficiently small, then $2 \pi+\mathcal{K}_{11}: C\left(S_{1}\right) \underset{1-1}{\text { onto }} C\left(S_{1}\right)$, and moreover, for all sufficiently large $N$,

$$
\left\|\left(2 \pi+\mathcal{P}_{1 N} \mathcal{K}_{11}\right)^{-1}\right\| \leq c<\infty .
$$

Using this, operate on (60) and (61) to obtain

$$
\begin{aligned}
& {\left[\begin{array}{cc}
I & \left(2 \pi+\mathcal{K}_{11}\right)^{-1} \mathcal{K}_{12} \\
\frac{1}{2 \pi} \mathcal{K}_{21} & I+\frac{1}{2 \pi} \mathcal{K}_{22}
\end{array}\right]\left[\begin{array}{l}
\rho_{1} \\
\rho_{2}
\end{array}\right]=\left[\begin{array}{c}
\left(2 \pi+\mathcal{K}_{11}\right)^{-1} g_{1} \\
\frac{1}{2 \pi} g_{2}
\end{array}\right]} \\
& {\left[\begin{array}{cc}
I & \left(2 \pi+\mathcal{P}_{1 N} \mathcal{K}_{11}\right)^{-1} \mathcal{P}_{1 N} \mathcal{K}_{12} \\
\frac{1}{2 \pi} \mathcal{P}_{2 N} \mathcal{K}_{21} & I+\frac{1}{2 \pi} \mathcal{P}_{2 N} \mathcal{K}_{22}
\end{array}\right]\left[\begin{array}{c}
\rho_{1 N} \\
\rho_{2 N}
\end{array}\right],} \\
& =\left[\begin{array}{c}
\left(2 \pi+\mathcal{P}_{1 N} \mathcal{K}_{11}\right)^{-1}\left(\mathcal{P}_{1 N} g\right)_{1} \\
\frac{1}{2 \pi}\left(\mathcal{P}_{2 N} g\right)_{2}
\end{array}\right]
\end{aligned}
$$

We write these equations in the simpler forms

$$
(I+\mathcal{H}) \tilde{\rho}=r, \quad\left(I+\mathcal{H}_{N}\right) \tilde{\rho}_{N}=r_{N},
$$

respectively, with $\tilde{\rho}=\left[\rho_{1}, \rho_{2}\right]^{T}, \tilde{\rho}_{N}=\left[\rho_{1 N}, \rho_{2 N}\right]^{T}$.

The operator $\mathcal{H}: \mathcal{X} \rightarrow \mathcal{X}$ is compact and the family $\left\{\mathcal{H}_{N}\right\}$ is a pointwise-convergent and collectively compact family, converging pointwise to $\mathcal{H}$. With the known invertibility of $2 \pi+\mathcal{K}$ on $C(S)$, we can obtain the invertibility of $I+\mathcal{H}$. Using the theory of collectively compact operator approximations, we have the existence and uniform boundedness of $\left(I+\mathcal{H}_{N}\right)^{-1}$ for all sufficiently large $N$; this leads directly to the result (57) asserted in the theorem. The result (58) follows from the identity (21) given earlier.

For convergence of the collocation solutions $\left\{\rho_{N}\right\}$, the standard result

$$
\left\|\rho-\rho_{N}\right\|_{\infty} \leq \frac{1}{2 \pi}\left\|\left(2 \pi+\mathcal{P}_{N} \mathcal{K}\right)^{-1}\right\|\left\|\rho-\mathcal{P}_{N} \rho\right\|_{\infty}
$$

implies $\left\|\rho-\rho_{N}\right\|_{\infty}=O\left(\widehat{\delta}_{N}^{3}\right)$ from the bound (13) for interpolation error. 
The condition (56) and the other assumptions of [23] on the solid angle are quite restrictive, and it is clear from the numerical examples that they are not necessary in practice. Other somewhat less restrictive assumptions on $S$ are given in [14], [15], [17]-[19], but for our proof of stability, we still require (56). Our results on rates of convergence assume only the stability results (57) and (58), and do not depend on how they are obtained. Other tools for proving stability are given in [10] and [12], and it may be possible to adapt them to our use of piecewise polynomial isoparametric interpolation. Again, they consider only polyhedral surfaces and thus do not need to approximate the surface.

We cannot show superconvergence of $\hat{\rho}_{N}$ at the node points (which was shown in Theorem 3.1 for $S$ a smooth surface.) For $S$ only piecewise smooth, $\mathcal{K}$ is no longer a smoothing operator, and that appears to prevent superconvergence.

5.1. Using the approximate surface. In practice, we solve the linear system (41), which uses the approximate surface $\widetilde{S}_{N}$. We also approximate the solid angle $\Omega(P)$ by the quantity $\Omega_{N}(P)$ defined in (43).

THEOREM 5.2. Let $S$ be a piecewise smooth surface, and let $P$ be a node point on $S$. Then

$$
\Omega(P)-\Omega_{N}(P)=O\left(\widehat{\delta}_{N}^{2}\right) .
$$

Proof. We first compute the error contributed by $\Delta_{K}$ which contains $P$. Without loss of generality, assume $P=m_{K}(0,0)$. Let

$$
P=\left(p_{1}, p_{2}, p_{3}\right)=m_{K}(0,0)=\tilde{m}_{K}(0,0) .
$$

We break error over $\Delta_{K}$ into two parts:

$$
\begin{aligned}
E_{1}=\int_{\sigma}\left[\left(D_{s} m_{K} \times D_{t} m_{K}\right) \cdot \frac{P-m_{K}(s, t)}{\left|P-m_{K}(s, t)\right|^{3}}\right. & \\
& \left.\quad-\left(D_{s} \tilde{m}_{K} \times D_{t} \tilde{m}_{K}\right) \cdot \frac{P-\tilde{m}_{K}(s, t)}{\left|P-m_{K}(s, t)\right|^{3}}\right] d s d t
\end{aligned}
$$

and

$$
\begin{aligned}
E_{2}=\int_{\sigma}\left[\left(D_{s} \tilde{m}_{K} \times D_{t} \tilde{m}_{K}\right) \cdot \frac{P-\tilde{m}_{K}(s, t)}{\left|P-m_{K}(s, t)\right|^{3}}\right. & \\
& \left.\quad-\left(D_{s} \tilde{m}_{K} \times D_{t} \tilde{m}_{K}\right) \cdot \frac{P-\tilde{m}_{K}(s, t)}{\left|P-\tilde{m}_{K}(s, t)\right|^{3}}\right] d \sigma .
\end{aligned}
$$

We now manipulate the first part of the integrand of (65).

$$
\begin{aligned}
& \left(D_{s} m_{K}(s, t) \times D_{t} m_{K}(s, t)\right) \cdot \frac{P-m_{K}(s, t)}{\left|P-m_{K}(s, t)\right|^{3}} \\
& \quad=\frac{\left(x_{s}^{2} x_{t}^{3}-x_{s}^{3} x_{t}^{2}, x_{s}^{3} x_{t}^{1}-x_{s}^{1} x_{t}^{3}, x_{s}^{1} x_{t}^{2}-x_{s}^{2} x_{t}^{1}\right) \cdot\left(p_{1}-x^{1}, p_{2}-x^{2}, p_{3}-x^{3}\right)}{\left[\left(p_{1}-x^{1}\right)^{2}+\left(p_{2}-x^{2}\right)^{2}+\left(p_{3}-x^{3}\right)^{2}\right]^{3 / 2}}
\end{aligned}
$$

Using the Taylor error formula for the $x^{i}$ about $(s, t)=(0,0)$, the numerator of equation (67) becomes

$$
\begin{aligned}
& \left(x_{s}^{2} x_{t}^{3}-x_{s}^{3} x_{t}^{2}, x_{s}^{3} x_{t}^{1}-x_{s}^{1} x_{t}^{3}, x_{s}^{1} x_{t}^{2}-x_{s}^{2} x_{t}^{1}\right) \cdot\left(p_{1}-x^{1}, p_{2}-x^{2}, p_{3}-x^{3}\right) \\
& =\left(x_{t}^{1} x_{s}^{2}-x_{t}^{2} x_{s}^{1}\right)\left(s^{2} x_{s s}^{3}+2 s t x_{s t}^{3}+t^{2} x_{t t}^{3}\right)+\left(x_{t}^{2} x_{s}^{3}-x_{t}^{3} x_{s}^{2}\right)\left(s^{2} x_{s s}^{1}+2 s t x_{s t}^{1}+t^{2} x_{t t}^{1}\right) \\
& \quad+\left(x_{t}^{3} x_{s}^{1}-x_{t}^{1} x_{s}^{3}\right)\left(s^{2} x_{s s}^{2}+2 s t x_{s t}^{2}+t^{2} x_{t t}^{2}\right)+O\left(\widehat{\delta}_{K}^{5}\right) .
\end{aligned}
$$


Computing the corresponding part of the second term of (65) with the same formula as we had above, we get

$$
\begin{aligned}
& \quad\left(D_{s} \tilde{m}_{K} \times D_{t} \tilde{m}_{K}\right) \cdot\left(P-\tilde{m}_{K}(s, t)\right) \\
& =\quad\left(x_{t}^{1} x_{s}^{2}-x_{t}^{2} x_{s}^{1}\right)\left(s^{2} x_{s s}^{3}+2 s t x_{s t}^{3}+t^{2} x_{t t}^{3}\right)+\left(x_{t}^{2} x_{s}^{3}-x_{t}^{3} x_{s}^{2}\right)\left(s^{2} x_{s s}^{1}+2 s t x_{s t}^{1}+t^{2} x_{t t}^{1}\right) \\
& \quad+\left(x_{t}^{3} x_{s}^{1}-x_{t}^{1} x_{s}^{3}\right)\left(s^{2} x_{s s}^{2}+2 s t x_{s t}^{2}+t^{2} x_{t t}^{2}\right)+O\left(\widehat{\delta}_{K}^{3}\right) .
\end{aligned}
$$

Thus,

$$
\left(D_{s} m_{K} \times D_{t} m_{K}\right) \cdot\left(P-m_{K}(s, t)\right)-\left(D_{s} \tilde{m}_{K} \times D_{t} \tilde{m}_{K}\right) \cdot\left(P-\tilde{m}_{K}(s, t)\right)=O\left(\widehat{\delta}_{K}^{5}\right) .
$$

Expanding each $x^{i}$ about $(0,0)$, the denominator of $(67)$ is

$$
\left[\left(p_{1}-x^{1}\right)^{2}+\left(p_{2}-x^{2}\right)^{2}+\left(p_{3}-x^{3}\right)^{2}\right]^{-3 / 2}=O\left(\left|\widehat{\delta}_{K}\right|^{-3}\right) .
$$

Then,

$$
\begin{aligned}
& \int_{\sigma} \frac{\left(D_{s} m_{K} \times D_{t} m_{K}\right) \cdot\left(P-m_{K}(s, t)\right)-\left(D_{s} \tilde{m}_{K} \times D_{t} \tilde{m}_{K}\right) \cdot\left(P-\tilde{m}_{K}(s, t)\right)}{\left|P-m_{K}(s, t)\right|^{3}} d \sigma \\
& =O\left(\widehat{\delta}_{K}^{2}\right) .
\end{aligned}
$$

Note there are at most six triangles containing the node point $P$, and the total error contributed from the $\Delta_{K}$ 's which contain $P$ is $O\left(\widehat{\delta}_{K}^{2}\right)$.

To analyze $E_{2}$, we need to know the error from the following:

$$
\begin{aligned}
& \left|\frac{1}{\left|P-m_{K}(s, t)\right|^{3}}-\frac{1}{\left|P-\widetilde{m}_{K}(s, t)\right|^{3}}\right| \\
& \leq\left|\frac{1}{\left|P-m_{K}(s, t)\right|}-\frac{1}{\left|P-\tilde{m}_{K}(s, t)\right|}\right| \cdot \mid \frac{1}{\left|P-m_{K}(s, t)\right|^{2}} \\
& \quad+\frac{1}{\left(\left|P-m_{K}(s, t)\right|\right)\left(\left|P-\tilde{m}_{K}(s, t)\right|\right.}+\frac{1}{\left|P-\tilde{m}_{K}(s, t)\right|^{2}} \mid \\
& \leq O\left(\widehat{\delta}_{K}\right) \cdot O\left(\widehat{\delta}_{K}^{-2}\right)=O\left(\widehat{\delta}_{K}^{-1}\right) .
\end{aligned}
$$

Then, from the above result and (68), we have the following error analysis for $E_{2}$ :

$$
\begin{aligned}
& \mid \int_{\sigma}\left(D_{s} \tilde{m}_{K} \times D_{t} \tilde{m}_{K}\right) \cdot\left(P-\tilde{m}_{K}(s, t)\right)\left[\frac{1}{\left|P-m_{K}(s, t)\right|^{3}}-\frac{1}{\left|P-\tilde{m}_{K}(s, t)\right|^{3}}\right] \\
& \quad \cdot d \sigma \mid \leq O\left(\widehat{\delta}_{K}^{4}\right) \cdot O\left(\widehat{\delta}_{K}^{-1}\right)=O\left(\widehat{\delta}_{K}^{3}\right) .
\end{aligned}
$$

Combining (69) and (70), we complete the proof of the first step, for $\Delta_{K}$ containing $P$.

Consider errors contributed by all $\Delta_{K}$ for which $P \notin \Delta_{K}$. Since $P \notin \Delta_{K}$, we can again treat the function $1 /\left|P-m_{K}(s, t)\right|^{3}$ as a smooth function. This proof will have two parts, as for that of Theorem 3.1, and we use results from the latter. Let $d_{K}, d$, and $r$ be the same as in Theorem 3.1.

Decompose the second part of proof as $E_{1}$ and $E_{2}$, the same as above in (65) and (67), respectively. In the previous part, we assumed that $P=m_{K}(0,0)$; and we now assume

$$
P \neq m_{K}(s, t) \quad \forall(s, t) \in \sigma
$$


Expand each $x^{i}$ about $(s, t)=(0,0)$ and compute

$$
\begin{aligned}
& \left(D_{s} m_{K} \times D_{t} m_{K}\right) \cdot\left(P-m_{K}(s, t)\right)-\left(D_{s} \tilde{m}_{K} \times D_{t} \tilde{m}_{K}\right) \cdot\left(P-\widetilde{m}_{K}(s, t)\right) \\
& \quad=E 4\left(s, t ; \widehat{v}_{2}-\widehat{v}_{1}, \widehat{v}_{3}-\widehat{v}_{1}\right)+E 5\left(s, t ; \widehat{v}_{2}-\widehat{v}_{1}, \widehat{v}_{3}-\widehat{v}_{1}\right)+O\left(\widehat{\delta}_{K}^{6}\right)
\end{aligned}
$$

$E 4$ is a homogeneous polynomial of degree three in $s$ and $t$, and its coefficients are $O\left(\widehat{\delta}_{K}^{4} \cdot d_{K}\right)$. Integrating $E 4$ over $\sigma$ yields zero. $E 5$ is also a polynomial in $s$ and $t$. Its coefficients are $O\left(\widehat{\delta}_{K}^{5}\right)$, and $E 5$ has the "odd function" property

$$
E 5\left(s, t ;-\left(\widehat{v}_{2}-\widehat{v}_{1}\right),-\left(\widehat{v}_{3}-\widehat{v}_{1}\right)\right)=-E 5\left(s, t ; \widehat{v}_{2}-\widehat{v}_{1}, \widehat{v}_{3}-\widehat{v}_{1}\right) \text {. }
$$

Thus, cancellation occurs.

When examining $E_{1}$, we need to expand the function $\left|P-m_{K}(s, t)\right|^{-3}$ about $(s, t)=$ $(0,0)$. Then we obtain

$$
\begin{aligned}
& \frac{\left(D_{s} m_{K} \times D_{t} m_{K}\right) \cdot\left(P-m_{K}(s, t)\right)-\left(D_{s} \tilde{m}_{K} \times D_{t} \tilde{m}_{K}\right) \cdot\left(P-\tilde{m}_{K}(s, t)\right)}{\left|P-m_{K}(s, t)\right|^{3}} \\
& =\frac{E 4+E 5+O\left(\widehat{\delta}_{K}^{6}\right)}{\left[\left(p_{1}-x^{1}(0,0)\right)^{2}+\left(p_{2}-x^{2}(0,0)\right)^{2}+\left(p_{3}-x^{3}(0,0)\right)^{2}\right]^{3 / 2}} \\
& \quad-\left(E 4+E 5+O\left(\widehat{\delta}_{K}^{6}\right)\right) e+O\left(\frac{\widehat{\delta}_{K}^{6}}{d_{K}^{4}}\right),
\end{aligned}
$$

where $e$ is a polynomial in $s$ and $t$ and its coefficients are $O\left(\widehat{\delta}_{K} / d_{K}^{4}\right)$. Integrating (71) over $\sigma$, the error contributed by each $\Delta_{K}$ is $O\left(\widehat{\delta}_{K}^{5} / d_{K}^{3}\right)+O\left(\widehat{\delta}_{K}^{6} / d_{K}^{4}\right)$. Then, using earlier methods, the global error $E_{1}$ can be shown to be $O\left(\hat{\delta}^{2}\right)$.

For computing $E_{2}$, we first calculate

$$
\begin{aligned}
& \left|\frac{1}{\left|P-m_{K}(s, t)\right|^{3}}-\frac{1}{\left|P-\tilde{m}_{K}(s, t)\right|^{3}}\right| \\
& \leq \quad\left|\frac{1}{\left|P-m_{K}(s, t)\right|}-\frac{1}{\left|P-\widetilde{m}_{K}(s, t)\right|}\right| \cdot \mid \frac{1}{\left|P-m_{K}(s, t)\right|^{2}} \\
& \quad+\frac{1}{\left(\left|P-m_{K}(s, t)\right|\right)\left(\left|P-\tilde{m}_{K}(s, t)\right|\right)}+\frac{1}{\left|P-\widetilde{m}_{K}(s, t)\right|^{2}} \mid .
\end{aligned}
$$

Using (40), (72) is $O\left(\widehat{\delta}_{K}^{3} / d_{K}^{2}\right) \cdot O\left(1 / d_{K}^{2}\right)=O\left(\widehat{\delta}_{K}^{3} / d_{K}^{4}\right)$. Therefore, the integrand of $E_{2}$ is

$$
\begin{aligned}
& \left|\left(D_{s} \tilde{m}_{K} \times D_{t} \tilde{m}_{K}\right) \cdot\left(P-\tilde{m}_{K}(s, t)\right)\left[\frac{1}{\left|P-m_{K}(s, t)\right|^{3}}-\frac{1}{\left|P-\tilde{m}_{K}(s, t)\right|^{3}}\right]\right| \\
& \quad \leq O\left(\widehat{\delta}_{K}^{2}\right) \cdot O\left(d_{K}\right) \cdot O\left(\frac{\widehat{\delta}_{K}^{3}}{d_{K}^{4}}\right)=O\left(\frac{\widehat{\delta}_{K}^{5}}{d_{K}^{3}}\right) .
\end{aligned}
$$

Thus, the error contributed by each $\Delta_{K}$ is $O\left(\widehat{\delta}_{K}^{5} / d_{K}^{3}\right)$, and $E_{2}$ is of order two. This proves the theorem. An almost identical proof also shows the result (49) used in the proof Theorem 3.5; we omit the details.

The above theorem shows the difference between the value of the solid angle and the approximate value of the solid angle. This result is not as good as desired. For smooth surfaces, the empirical rate seems to be $O\left(\widehat{\delta}_{N}^{3}\right)$, from the example of Table 1 and other similar 
examples. But the empirical results for piecewise smooth surfaces, given in the following section, do not clearly indicate a convergence rate.

THEOREM 5.3. Let $S$ be a piecewise smooth surface, satisfying the assumptions of $\S 2$, the hypotheses of [23] as descried earlier, and the solid angle assumption (56). Let $\rho$ be a solution of (2) or (5), with $\rho \in C(S) \cap C^{4}\left(S_{j}\right), j=1, \ldots, J$. Let $\tilde{\rho}_{N}$ be the solution of the system (41) which uses the approximation $\widetilde{S}_{N}$ for $S$. Then

$$
\max _{1 \leq i \leq N_{v}}\left|\rho\left(v_{i}\right)-\tilde{\rho}_{N}\left(v_{i}\right)\right|=O\left(\widehat{\delta}_{N}^{2}\right) .
$$

Proof. This is proven by combining the techniques used in the proof of Theorem 3.5, together with the results of Theorem 5.1 for the collocation method for (2) or (5) when the original surface $S$ is used. We omit the details

6. Numerical examples: Piecewise smooth surface case. The collocation integrals in the linear system (41) were evaluated with the same type of numerical integration used when $S$ was a smooth surface. The two-grid iteration method for solving the linear system required a modification from that used for the smooth surface case, and this is explored in [6]. Below we give examples for several piecewise smooth surfaces to empirically study the rate of convergence of the projection method and illustrate some of the results of $\S 5$. Since we are solving (5), we replace $\rho$ with $u$.

Note that in our examples, the true solutions $u(P)$ are all smooth functions when $P$ is off the boundary, and they are piecewise smooth on the boundary. In contrast, the presence of a piecewise smooth boundary usually leads to solutions that are ill behaved in a neighborhood of all edges and corners of the surface $S$. To deal with such solutions, a graded triangular mesh is needed. A theory describing the form of grading needed for Galerkin's method has been developed recently in [10]; a theory for collocation methods is described in [17]. These results are all limited to polyhedral boundaries.

6.1. The surfaces. We describe three surfaces, two of which are polyhedral. The first surface $(S \# 1)$ is an elliptical paraboloid with a cap:

$$
\begin{array}{ll}
\left(\frac{x}{a}\right)^{2}+\left(\frac{y}{b}\right)^{2}=z, & 0 \leq z \leq c, \\
\left(\frac{x}{a}\right)^{2}+\left(\frac{y}{b}\right)^{2} \leq c, & z=c .
\end{array}
$$

The second surface $(S \# 2)$ is the tetrahedron

$$
\left\{(x, y, z) \mid \frac{x}{a}+\frac{y}{b}+\frac{z}{c} \leq 1 ; x, y, z \geq 0\right\} .
$$

The third surface $(S \# 3)$ is an " $L$-block," described as follows. Define

$$
\begin{aligned}
L_{0}= & {[0,1] \times[0,2] \cup[0,2] \times[0,1] \subset \mathbf{R}^{2}, } \\
& D_{0}=[0,1] \times L_{0} \subset \mathbf{R}^{3}, \\
D= & \left\{(a x, b y, c z) \mid(x, y, z) \in D_{0}\right\},
\end{aligned}
$$

and let $S$ be the boundary of $D$. With all three surfaces, the constants $a, b$, and $c$ are positive.

Surface \#1 was chosen to illustrate the use of a curved surface, so that the use of the interpolatory approximate surface $\widetilde{S}$ would be nontrivial. Surface \#2 encloses a convex region and all boundary points satisfy the hypotheses of [23] although the angle assumption (56) is 
TABLE 6

Solid angle approximations on an elliptical paraboloid at selected $v_{i}$.

\begin{tabular}{rcrrrr}
\hline$i$ & $\Omega\left(v_{i}\right)$ & \multicolumn{1}{c}{$E 1$} & \multicolumn{1}{c}{$E 2$} & \multicolumn{1}{c}{$E 3$} & \multicolumn{1}{c}{$E 4$} \\
\hline 1 & $2 \pi$ & $2.35 \mathrm{E}-3$ & $-8.43 \mathrm{E}-6$ & $1.52 \mathrm{E}-8$ & $-2.30 \mathrm{E}-8$ \\
2 & $\pi / 2$ & $1.28 \mathrm{E}-1$ & $1.99 \mathrm{E}-2$ & $9.08 \mathrm{E}-3$ & $3.33 \mathrm{E}-4$ \\
6 & $2 \pi$ & $1.92 \mathrm{E}-2$ & $-7.82 \mathrm{E}-7$ & $1.16 \mathrm{E}-8$ & $-8.07 \mathrm{E}-8$ \\
7 & $2 \pi$ & $6.51 \mathrm{E}-1$ & $5.69 \mathrm{E}-2$ & $-3.95 \mathrm{E}-4$ & $-2.59 \mathrm{E}-3$ \\
8 & $\pi / 2$ & $2.56 \mathrm{E}-1$ & $1.59 \mathrm{E}-1$ & $4.08 \mathrm{E}-2$ & $1.00 \mathrm{E}-2$ \\
15 & $2 \pi$ & $1.69 \mathrm{E}-1$ & $-1.72 \mathrm{E}-4$ & $4.80 \mathrm{E}-8$ & $1.38 \mathrm{E}-7$ \\
\hline
\end{tabular}

not satisfied. Surface \#3 is also polyhedral, but now it encloses a nonconvex region. Moreover, some of the angles on the surface do not satisfy the assumption V3 of [23]. (V3 states that at each point of the boundary, either the interior or the exterior tangent cone must be convex.) For example, the tangent cones at $(x, y, z)=(0,1,1)$ and $(1,1,1)$ do not satisfy V3. Working with $S \# 3$ allows us to test whether or not the assumption V3 is necessary empirically. However, this surface does satisfy the assumptions of [14], which extends the earlier results of [23] to a slightly larger class of surfaces, albeit in a modified function space.

6.2. The solid angle. We again use the approximation (43) to approximate the interior solid angle $\Omega(v)$ at points $v \in S$, thus forcing all rows of the coefficient matrix for the linear system (41) to equal $4 \pi$. Results for an elliptical paraboloid $(S \# 1)$ are given in Table 6 at the following representative nodes:

$$
\begin{array}{lll}
v_{1}=(0,0,0), & v_{2}=(2,0,1), & v_{6}=(0,0,1), \\
v_{7}=(1,0, .25), & v_{8}=(\sqrt{2}, \sqrt{2}, 1), & v_{15}=(1,0,1) .
\end{array}
$$

The parameters used for the surface were $(a, b, c)=(2,2,1)$; and the integration parameters were $N_{g}=10$ and $N_{d}=0,1,2,2$ for $N=8,32,128,512$, respectively.

In Table 6, some of the entries have a rapid decrease in size as $N$ increases, and then the error stops decreasing and remains around $10^{-7}$ to $10^{-8}$. It seems likely that the latter is due to the limited accuracy in the numerical integration method, although we have not tested this. In general, there appears to be no pattern to the rate at which the error decreases. The case of $\Omega\left(v_{8}\right)$ is of interest since the error in this case is much larger than for the other cases, again for unknown reasons. According to Theorem 5.2, the errors should converge with a rate of at least $O\left(\widehat{\delta}_{N}^{2}\right)$, but this seems to be the case only for the node $v_{8}$.

The results for a polyhedral surface were much better. Table 7 contains results for the $L$-block $(S \# 3)$ at the following representative nodes:

$$
\begin{array}{lll}
v_{1}=(0,0,0), & v_{7}=(0,0,1), & v_{9}=(0,1,1), \\
v_{17}=(.5,0,1.5), & v_{20}=(.5,0,1.5), & v_{33}=(.5,1,1) .
\end{array}
$$

There is no approximation of the surface in this case, and thus all errors are due to the numerical integration being used. The resulting errors are very small.

The parameters for the $L$-block are $(a, b, c)=(1,1,1)$; for the integration parameters, we used $N_{d}=0,1,2$ for $N=28,112,448$, respectively. Note that in this case, there are no singular integrals because the double-layer kernel function $K(P, Q)$ is identically zero when $P$ and $Q$ belong to the same planar surface. The columns $E 1, E 2$, and $E 3$ denote the errors at the given $v_{i}$, for $N=28,112,448$, respectively.

6.3. Solution of the exterior Neumann problem. We begin with the solution of (3) for the elliptical paraboloid (S\#1). The problem (3) was solved with the normal derivative $f$ 
TABLE 7

Solid angle approximations on an L-block at selected $v_{i}$.

\begin{tabular}{rcrrr}
\hline$i$ & $\Omega\left(v_{i}\right)$ & \multicolumn{1}{c}{$E 1$} & \multicolumn{1}{c}{$E 2$} & \multicolumn{1}{c}{$E 3$} \\
\hline 1 & $\pi / 2$ & $-9.81 \mathrm{E}-5$ & $-2.39 \mathrm{E}-6$ & $-2.30 \mathrm{E}-8$ \\
7 & $\pi$ & $3.59 \mathrm{E}-4$ & $3.95 \mathrm{E}-6$ & $3.40 \mathrm{E}-8$ \\
9 & $3 \pi / 2$ & $5.60 \mathrm{E}-4$ & $5.53 \mathrm{E}-6$ & $4.10 \mathrm{E}-8$ \\
17 & $2 \pi$ & $1.88 \mathrm{E}-2$ & $2.88 \mathrm{E}-6$ & $-4.42 \mathrm{E}-7$ \\
20 & $2 \pi$ & $-1.87 \mathrm{E}-2$ & $1.03 \mathrm{E}-6$ & $-3.72 \mathrm{E}-7$ \\
33 & $3 \pi$ & $-3.36 \mathrm{E}-2$ & $1.01 \mathrm{E}-5$ & $-6.68 \mathrm{E}-7$ \\
\hline
\end{tabular}

TABLE 8

Maximum errors on an elliptical paraboloid.

\begin{tabular}{rcccc}
\hline \multicolumn{1}{c}{$N$} & $\left\|u_{1}-u_{1 N}\right\|_{\infty}$ & Ratio & $\left\|u_{2}-u_{2 N}\right\|_{\infty}$ & Ratio \\
\hline 8 & $2.89 \mathrm{E}-2$ & & $6.87 \mathrm{E}-2$ & \\
32 & $7.26 \mathrm{E}-3$ & 3.98 & $1.32 \mathrm{E}-2$ & 5.23 \\
128 & $2.75 \mathrm{E}-3$ & 2.65 & $4.47 \mathrm{E}-3$ & 2.94 \\
512 & $8.73 \mathrm{E}-4$ & 3.15 & $1.46 \mathrm{E}-3$ & 3.06 \\
\hline
\end{tabular}

TABLE 9

Errors $u_{1}-u_{1 N}$ at the selected points (74) on an elliptical paraboloid.

\begin{tabular}{rcccccc}
\hline \multicolumn{1}{c}{$i$} & $E 2$ & $E 3$ & $E 4$ & $E 1 / E 2$ & $E 2 / E 3$ & $E 3 / E 4$ \\
\hline 1 & $-6.50 \mathrm{E}-3$ & $-2.12 \mathrm{E}-3$ & $-4.87 \mathrm{E}-4$ & 4.45 & 3.07 & 4.35 \\
2 & $-1.62 \mathrm{E}-3$ & $-3.16 \mathrm{E}-4$ & $-4.01 \mathrm{E}-5$ & 1.49 & 5.13 & 7.88 \\
6 & $-7.26 \mathrm{E}-3$ & $-1.21 \mathrm{E}-3$ & $-1.39 \mathrm{E}-4$ & 1.07 & 5.99 & 8.71 \\
7 & $-6.05 \mathrm{E}-3$ & $-9.28 \mathrm{E}-4$ & $-1.19 \mathrm{E}-4$ & 2.92 & 6.16 & 8.23 \\
8 & $-2.45 \mathrm{E}-3$ & $-4.42 \mathrm{E}-4$ & $-5.69 \mathrm{E}-5$ & 1.75 & 5.53 & 7.76 \\
15 & $-5.23 \mathrm{E}-3$ & $-8.15 \mathrm{E}-4$ & $-9.19 \mathrm{E}-5$ & 0.80 & 6.42 & 8.87 \\
\hline
\end{tabular}

chosen from the true solution $u$. The two cases used were

$$
u_{1}(x, y, z)=\frac{1}{r}, \quad u_{2}(x, y, z)=\frac{1}{r} \exp \left(x / r^{2}\right) \cos \left(\left(z-\frac{1}{2} c\right) / r^{2}\right),
$$

with $r=\left|(x, y, z)-\left(0,0, \frac{1}{2} c\right)\right|$.

Table 8 contains the maximum errors at the node points, for $(a, b, c)=(2,2,2)$. The integration parameters used were $N_{g}=10$ and $N_{d}=0,1,2,2$ for $N=8,32,128,512$, respectively. To better understand the behavior of the error, Table 9 contains the errors for $u_{1 N}$ at the following representative vertices $v_{i}$ :

$$
\begin{array}{lll}
v_{1}=(0,0,0), & v_{2}=(\sqrt{8}, 0,2), & v_{6}=(0,0,2), \\
v_{7}=(\sqrt{2}, 0, .5), & v_{8}=(2,2,2), & v_{15}=(\sqrt{2}, 0,2) .
\end{array}
$$

The nodes $v_{2}$ and $v_{8}$ are on the edge at $z=2$, the nodes $v_{1}$ and $v_{8}$ are on the lateral subsurface, and the nodes $v_{6}$ and $v_{15}$ are in the interior of the top subsurface. Again, the notation $E 1, E 2$, $E 3, E 4$ denotes the error for $N=8,32,128,512$, respectively.

On the basis of Table 8, the rate of convergence might be either $O\left(\widehat{\delta}_{N}\right)$ or $O\left(\widehat{\delta}_{N}^{2}\right)$, although Theorem 5.3 implies that the order of convergence should be at least $O\left(\widehat{\delta}_{N}^{2}\right)$ when the true solution $u(P)$ is a smooth function on each smooth section of the surface $S$. By examining the errors given in Table 9 at a representative set of node points, it seems likely that the order of convergence for $\left\|u-u_{N}\right\|_{\infty}$ is higher, probably $O\left(\widehat{\delta}_{N}^{3}\right)$.

We give results for the simplex $S \# 2$, with $(a, b, c)=(3,3,3)$. The Neumann data $f$ was chosen from the true solutions

$$
u_{1}(x, y, z)=\frac{1}{r}, \quad u_{2}(x, y, z)=\frac{1}{r} \exp \left(\left(x-\frac{1}{4} a\right) / r^{2}\right) \cos \left(\left(z-\frac{1}{4} c\right) / r^{2}\right),
$$


TABLE 10

Maximum errors on a simplex.

\begin{tabular}{rcccc}
\hline \multicolumn{1}{c}{$N$} & $\left\|u_{1}-u_{1 N}\right\|_{\infty}$ & Ratio & $\left\|u_{2}-u_{2 N}\right\|_{\infty}$ & Ratio \\
\hline 4 & $4.49 \mathrm{E}-1$ & & 2.17 & \\
16 & $2.16 \mathrm{E}-2$ & 20.8 & $7.50 \mathrm{E}-1$ & 2.89 \\
64 & $1.08 \mathrm{E}-2$ & 2.00 & $1.06 \mathrm{E}-1$ & 7.08 \\
256 & $5.79 \mathrm{E}-4$ & 18.6 & $1.96 \mathrm{E}-2$ & 5.41 \\
\hline
\end{tabular}

TABLE 11

Maximum errors on an L-block.

\begin{tabular}{rcccc}
\hline \multicolumn{1}{c}{$N$} & $\left\|u_{1}-u_{1 N}\right\|_{\infty}$ & Ratio & $\left\|u_{2}-u_{2 N}\right\|_{\infty}$ & Ratio \\
\hline 28 & $2.58 \mathrm{E}-2$ & $6.10 \mathrm{E}-1$ & & \\
112 & $1.53 \mathrm{E}-3$ & 16.9 & $7.56 \mathrm{E}-2$ & 8.07 \\
448 & $2.10 \mathrm{E}-4$ & 7.29 & $3.69 \mathrm{E}-3$ & 20.5 \\
\hline
\end{tabular}

TABLE 12

Errors in $u_{1 N}\left(v_{i}\right)$ at representative points on L-block.

\begin{tabular}{rrrrr}
\hline \multicolumn{1}{c}{$\boldsymbol{c}$} & \multicolumn{1}{c}{$E 1$} & \multicolumn{1}{c}{$E 2$} & \multicolumn{1}{c}{$E 3$} & $E 2 / E 3$ \\
\hline 1 & $-7.51 \mathrm{E}-3$ & $-7.71 \mathrm{E}-5$ & $-3.27 \mathrm{E}-6$ & 23.6 \\
7 & $-8.49 \mathrm{E}-4$ & $-7.75 \mathrm{E}-5$ & $-4.19 \mathrm{E}-7$ & 185.0 \\
9 & $-9.83 \mathrm{E}-3$ & $-3.82 \mathrm{E}-4$ & $-1.70 \mathrm{E}-5$ & 22.6 \\
17 & $-1.11 \mathrm{E}-3$ & $2.58 \mathrm{E}-4$ & $2.06 \mathrm{E}-5$ & 12.6 \\
20 & $7.22 \mathrm{E}-4$ & $3.88 \mathrm{E}-4$ & $3.06 \mathrm{E}-5$ & 12.7 \\
33 & $-2.58 \mathrm{E}-2$ & $-1.53 \mathrm{E}-3$ & $-9.26 \mathrm{E}-5$ & 16.5 \\
\hline
\end{tabular}

with $r=\left|(x, y, z)-\frac{1}{4}(a, b, c)\right|$. The integration parameter used was $N_{d}=0,1,2,3$ for $N=4,16,64,256$, respectively. No singular integrations were needed because the surface was piecewise planar for the reasons discussed above in connection with the computation of the solid angle for the $L$-block.

The results are given in Table 10. After analyzing them, one can say only that the order of convergence seems to be at least $O\left(\widehat{\delta}_{N}^{2}\right)$. From Theorem 5.1, the error in this case is $O\left(\widehat{\delta}_{N}^{3}\right)$, provided the stability result (57) is known to be true.

The third set of examples is for the $L$-block, with $(a, b, c)=(1,1,1)$. The true solution used is

$$
u_{1}(x, y, z)=\frac{1}{r}, \quad u_{2}(x, y, z)=\frac{1}{r} \exp \left(\left(x-\frac{1}{2} a\right) / r^{2}\right) \cos \left(\left(z-\frac{1}{2} c\right) / r^{2}\right),
$$

with $r=\left|(x, y, z)-\frac{1}{2}(a, b, c)\right|$. The integration parameter used was $N_{d}=0,1,2$ for $N=28,112,448$, respectively. No singular integrations were needed because the surface was piecewise planar, for the reasons discussed earlier. The maximum errors at the node points are given in Table 11. Table 12 lists the errors at the individual nodes of (73) to give a more complete picture of the behavior of the error. The quantities $E 1, E 2, E 3$ represent the error for $N=28,112,448$, respectively.

From Theorem 5.1, the error in this case is $O\left(\widehat{\delta}_{N}^{3}\right)$, provided the stability result (57) is known to be true. The errors in Table 11 are insufficient to predict an order of convergence, although it appears to be $O\left(\widehat{\delta}_{N}^{3}\right)$ or faster. From Table 12, the errors appear to be of order $O\left(\widehat{\delta}_{N}^{4}\right)$, if one is to choose an integer power for the order. Recall that this surface does not satisfy the assumption V3 of [23] (the point $v_{9}$ violates the assumption). Clearly, our results indicate that this assumption is an artifact of the method of proof and is unnecessary in practice. 


\section{REFERENCES}

[1] K. AtKinson, A Survey of Numerical Methods for the Solution of Fredholm Integral Equations of the Second Kind, Society for Industrial and Applied Mathematics, Philadelphia, 1976.

[2] - Piecewise polynomial collocation for integral equations on surfaces in three dimensions, J. Integral Equations Appl., 9 (suppl.) (1985), pp. 25-48.

[3] - Solving integral equations on surfaces in space, in Constructive Methods for the Practical Treatment of Integral Equations, G. Hämmerlin and K. Hoffman, eds., Birkhäuser, Basel, 1985, pp. 20-43.

[4] - An empirical study of the numerical solution of integral equations on surfaces in $\mathbf{R}^{3}$, Reports on Computational Mathematics, \#1, Dept. of Mathematics, University of Iowa, Iowa City, IA, 1989.

[5] - A survey of boundary integral equation methods for the numerical sollution of Laplace's equation in three dimensions, in Numerical Solution of Integral Equations, M. Golberg, ed., Plenum Press, New York, 1990, pp. 1-34.

[6] - Two-grid iteration methods for linear integral equations of the second kind on piecewise smooth surfaces in $\mathbf{R}^{3}$, SIAM J. Sci. Comp., 15 (1994), pp. 1083-1104.

[7] K. ATKINSON AND I. GRAHAM, Iterative solution of linear systems arising from the boundary integral method, SIAM J. Sci. Statis. Comput., 13 (1992), pp. 694-722.

[8] D. CHIEN, Piecewise Polynomial Collocation for Integral Equations on Surfaces in Three Dimensions, Ph.D. thesis, University of Iowa, Iowa City, IA, 1991.

[9] M. DUFFY, Quadrature over a pyramid or cube of integrands with a singularity at the vertex, SIAM J. Numer. Anal., 19 (1982), pp. 1260-1262.

[10] J. ElSCHNER, The double-layer potential operator over polyhedral domains II. Spline Galerkin methods, Math. Methods Appl. Sci., 15 (1992), pp. 23-37.

[11] G. FaIRWEATHER, F. RIzZO, AND D. SHIPPY, Computation of double integrals in the boundary integral method, in Advances in Computer Methods for Partial Differential Equations-III, R. Vichnevetsky and R. Stepleman, eds., IMACS Symposium, Rutgers University, Brunswick, New Jersey, 1979, pp. 331-334.

[12] I. GRAhAm AND G. ChANDLER, High order methods for linear functionals of solutions of second kind integral equations, SIAM J. Numer. Anal., 25 (1988), pp. 1118-1137.

[13] C. JOHNSON AND L. SCOTT, An analysis of quadrature errors in second-kind boundary integral equations, SIAM J. Numer. Anal., 26 (1989), pp. 1356-1382.

[14] J. KRAL AND W. WENDLAND, Some examples concerning applicability of the Fredholm-Radon method in potential theory, Aplicace Matem., 31 (1986), pp. 293-308.

[15] - On the applicability of the Fredholm-Radon method in potential theory and the panel method, in Panel Methods in Fluid Mechanics with Emphasis in Aerodynamics, J. Ballmann, R. Eppler, W. Hackbusch, eds., Vieweg, Braunschweig, 1988, pp. 120-136.

[16] S. Mikhlin, Mathematical Physics: An Advanced Course, North-Holland, Amsterdam, 1970.

[17] A. RATHSFELD, On quadrature methods for the double layer potential equation over the boundary of a polyhedron. Numer. Math., 66 (1993), pp. 67-95.

[18] The invertibility of the double-layer potential operator on the space of continuous functions defined on a polyhedron: The panel method, Applicable Anal., 45 (1992), pp. 135-177.

[19] - Iterative solution of linear systems arising from the Nyström method for the double-layer potential equation over curves with corners, Math. Methods Appl. Sci., 16 (1993), pp. 443-455.

[20] J. RAdon, Über die Randwertaufgaben beim logarithmischen Potential, Sitzungsberichte der Akademie der Wissenschafter Wien, 128 Abt. IIa (1919), pp. 1123-1167.

[21] C. SCHWAB AND W. WENDLAND, On numerical cubatures of singular surface integrals in boundary element methods, Numerische Math., 62 (1992), pp. 343-369.

[22] A. Stroud, Approximate Calculation of Multiple Integrals, Prentice-Hall, Englewood Cliffs, NJ, 1971.

[23] W. WENDLAND, Die Behandlung von Randwertaufgaben im $\mathbf{R}^{3}$ mit Hilfe von Einfach- und Doppelschichtpotentialen, Numer. Math., 11 (1968), pp. 380-404. 